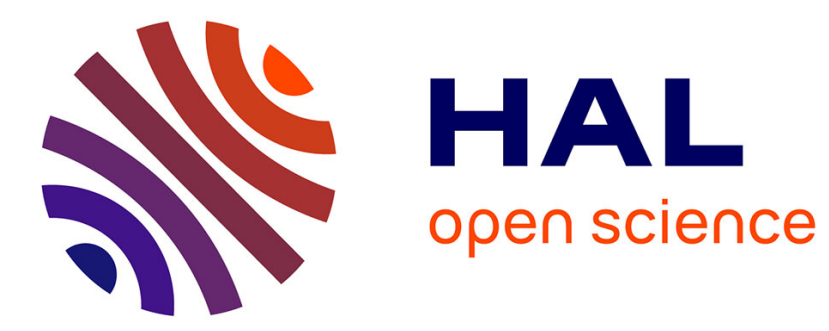

\title{
Mutual Service Processes in Euclidean Spaces: Existence and Ergodicity
}

François Baccelli, Fabien Mathieu, Ilkka Norros

\section{To cite this version:}

François Baccelli, Fabien Mathieu, Ilkka Norros. Mutual Service Processes in Euclidean Spaces: Existence and Ergodicity. Queueing Systems, 2017, 86 (1-2), pp.95 - 140. 10.1007/s11134-017-95243. hal-01535925

\section{HAL Id: hal-01535925 \\ https://hal.inria.fr/hal-01535925}

Submitted on 9 Jun 2017

HAL is a multi-disciplinary open access archive for the deposit and dissemination of scientific research documents, whether they are published or not. The documents may come from teaching and research institutions in France or abroad, or from public or private research centers.
L'archive ouverte pluridisciplinaire HAL, est destinée au dépôt et à la diffusion de documents scientifiques de niveau recherche, publiés ou non, émanant des établissements d'enseignement et de recherche français ou étrangers, des laboratoires publics ou privés. 


\title{
Mutual Service Processes in Euclidean Spaces: Existence and Ergodicity
}

\author{
François Baccelli, Fabien Mathieu, Ilkka Norros \\ UT Austin, USA, Nokia Bell Labs, France, VTT, Finland
}

June 9, 2017

\begin{abstract}
Consider a set of objects, abstracted to points of a spatially stationary point process in $\mathbb{R}^{d}$, that deliver to each other a service at a rate depending on their distance. Assume that the points arrive as a Poisson process and leave when their service requirements have been fulfilled. We show how such a process can be constructed and establish its ergodicity under fairly general conditions. We also establish a hierarchy of integral balance relations between the factorial moment measures and show that the time-stationary process exhibits a repulsivity property.
\end{abstract}

AMS Classification: 60D05, 60G55, 60D05, 60G10, 60G17, 60J25, 05C80, 70F45.

Keywords: spatial birth and death process, infinite particle system, Palm probability, coupling from the past, moment measure, repulsion.

\section{Introduction}

This paper was initially motivated by our study of peer to peer file-sharing in spatial scenarios, where the transmission speed between two peers depends on their distance [1]. The peers ${ }^{1}$ join as a Poisson rain in $\mathbb{R}^{2}$, serve each other at rates given by some decreasing function $f$ of their distances, and leave when their individual service requirements (assumed to be exponentially distributed, i.e. memoryless) have been fulfilled.

More precisely, all peers aim at getting some given file. There is a flow of peers arriving to get the file, and leaving as soon as they get it. All peers present in the system at any time echange small pieces of the file on a tit for tat basis. This exchange mechanism leads to the service process described above, see [1].

In [1], assuming that an ergodic steady state of the process exists in $\mathbb{R}^{2}$, we derived many interesting properties and formulae related to the superscalability of such mutual service systems: the larger the arrival rate, the shorter the mean service time experienced by a peer. Proving the existence of a time and space stationary and ergodic

\footnotetext{
${ }^{1}$ By 'peers' we mean network nodes that are able to make direct contacts to other nodes.
} 
regime turns out to be difficult because of (a) the non-monotonicity of the dynamics: when a peer leaves, it also ceases to serve the others; and (b) the fact that unbounded rate functions are necessary within this context, which prevents the use of classical approaches. The present paper solves this existence issue using coupling techniques and lays the ground for further studies on this class of models.

We consider a class of motion invariant ${ }^{2}$ spatial birth and death processes in $\mathbb{R}^{d}$, where the births take place according to a homogeneous Poisson rain and where the instantaneous death rate of a point of the current configuration is the shot-noise [21] of the configuration at this point for some positive response function $f$. This class of processes features non-trivial interactions between points combining "density" and "geometry" components that may have many other practically important incarnations. This comes from the fact that the death rate cannot be assessed through densities only, as the connections are also functions of distances. In fact, the presence of a point at some location implies that there are less points around than what a density argument would predict. This Palm type bias [2] makes the role of geometry quite central.

Our main results are (i) a proof of the existence and uniqueness of a stationary regime (Theorem 7.4); (ii) a hierarchy of balance equations linking the factorial moment measures [2] of neighboring orders (Theorem 8.1) for this problem, generalizing those conjectured in [1] for discussing superscalability; (iii) a general repulsion result formalizing the Palm bias alluded to above (Theorem 8.2); (iv) a result on the exponential speed at which the initial condition is forgotten (Theorem 7.1).

Our work can be related to at least three approaches in existing literature. First, Garcia and Kurtz have studied spatial birth and death processes on $\mathbb{R}^{d}$ using several techniques, e.g. the construction of rather general processes as projections of higherdimensional Poisson processes [8, 10] and stochastic equations [9].

Second, Penrose [19] has proposed a general method for proving the existence and the uniqueness of the stationary regime of spatial birth and death processes. The idea is to represent the point process in continuum as a family of processes indexed by a graph with bounded neighborhood size. The Markov approach discussed in this reference can be applied to a variety of dynamics and particle systems beyond spatial birth and death processes. It also leads to SLLN and CLT theorems.

Third, an approach using strong functional analytic techniques has been developed by Kondratiev and Finkelshtein (with co-authors, see e.g. [14, 4, 5]). For instance, [5] studies spatial birth and death processes with birth and death rates determined by a shot-noise process of the current configuration. The authors study the evolution of the correlation functions of the process as elements of a Banach space and show that this uniquely determines the evolution of the distribution of the spatial birth and death process on certain compact sets of time. They also develop conservation equations for higher order moment measures [6,4]. The model studied in our paper appears in, e.g., [3] and [13], and the latter also gives a proof of the existence of an evolution starting at time 0 .

In comparison to the above references, our main novelties/differences are the fol-

\footnotetext{
${ }^{2}$ By motion invariant, we mean invariance w.r.t. all translations and rotations of $\mathbb{R}^{d}$.
} 
lowing:

(1) On finite time intervals, our algorithm Construct presents a constructive probabilistic alternative to the projection construction of $[8,10]$, to the Markov construction of [19] and to the semi-group construction of [5]; more importantly, our approach does not require the boundedness assumptions which are made in the rate functions of [19] and [8] (Condition (3.1)), and in the response function of [5]. The model considered in the present paper requires handling unbounded death rates (this is inherent to shot noise fields) and also covers interactions with unbounded support.

(2) This probabilistic approach extends to the joint construction of the evolutions associated with two different initial conditions in terms of an explicit non-trivial coupling in the extended algorithm $\left(\right.$ Construct $\left.^{Z}\right)$, which allows one to build the evolutions with two initial conditions jointly.

(3) This coupling is combined with the differential equations governing the time evolution of Palm expectations of the death rates experienced by different classes of points, related to the moment measure method alluded to above. The main novelty here is in the way this is used to prove an exponential convergence result on densities (Theorem 7.1) and to derive computational estimates and bounds on densities (Section 8.2), as well as structural properties on the stationary regime (like repulsion).

(4) This coupling is also instrumental in the construction of the stationary regime in terms of a coupling from the past, discussed in Subsection 7.2, also new to the best of our knowledge.

(5) Finally, the coupling allows one to consider time and space ergodicity properties that are not much discussed in the references above (one exception being [8] in the bounded rate function case).

Although the present paper is strictly focusing on the case of shot-noise deaths, its ideas may turn out to be valuable also for studying other non-monotonous infinite geometric particle systems. Future research directions directly related to the present paper include the relaxation of certain assumptions on $f$, the case of non-exponential service requirement distribution, the "fluid" and "hard-core" limit regimes considered in [1], and multi-chunk models.

The paper is structured as follows. Section 2 specifies the model from two points of view and details the assumptions on $f$. The next sections focus on the construction of the stationary regime. If the phase space were compact, one could use the formalism of $[20,11,17]$ together with Markovian techniques, but our first challenge is to construct the process on $\mathbb{R}^{d}$. Section 3 proposes a pathwise construction through an infinitely running algorithm called Construct. The second step, presented in Section 4, is to build a coupling between the dynamics with an empty initial state and that with a motion invariant initial state $\mathcal{Z}_{0}$. The coupling is constructed pathwise by the algorithm 
Construct $^{Z}$, a natural extension of Construct. Martingale and random walk arguments show that the influence of each individual point of $\mathcal{Z}_{0}$ dies out in finite time. This is, however, not yet sufficient for establishing ergodicity. Section 5 notes useful tightness and positivity properties of the system. Combining these with differential equations describing the time evolution, we then prove in Section 6 that on any compact set of space, the time of last influence of $\mathcal{Z}_{0}$ is actually integrable. This finally allows to develop a coupling from the past argument in Section 7, proving the fundamental existence and ergodicity result of the paper. Finally, Section 8 gathers some basic properties of the stationary distribution of the system. The differential equations alluded to above lead to a set of conservation laws for moment measures that mimic the Markov birth and death structure.

The appendix Section 9 details the proofs of some of the equations. It also provides a table of notation. For all classical point process theory results used in the paper (e.g. Palm probabilities, higher order factorial moment measures, Campbell's theorem, Slivnyak's theorem), the reader is invited to consult [2].

\section{Model}

We start with two informal definitions of the stochastic process of interest. We then give a formal definition of the problem.

\subsection{Model description}

\subsubsection{Spatial birth and death viewpoint}

Let $D$ be a closed convex set in $\mathbb{R}^{d}$. Let $M(D)$ denote the set of counting measures $\phi$ on $D$ (see e.g. [12]). Depending on the situation, we will consider the point process $\phi$ either as a counting measure, or as a set, the support of this counting measure. As a result, the number of points of $\phi$ in the Borel set $C$ will be denoted either $\phi(C)$ or $|\phi \cap C|$, with $|S|$ the cardinality of the set $S$, depending on the circumstances. Let $\mathcal{M}(D)$ denote the smallest $\sigma$-field containing all the events $\phi(C)=k, C$ ranging over bounded Borel subsets of $D$ and $k$ over integers.

We consider a spatial birth and death (SBD) process on $D$, namely a $M(D)$-valued Markov jump process [20]. The state (or point configuration) of this Markov process at time $t$ will be denoted by $\Phi_{t} \in M(D)$.

When $D$ is compact, such a Markov process is characterized by two rate functionals, the birth rate functional $b\left(\phi, \phi+\delta_{x}\right)$, which gives the infinitesimal rate of a birth at $x \in D$ in configuration $\phi \in M(D)$ and the death rate functional $\mu\left(\phi+\delta_{x}, \phi\right)$, which gives the infinitesimal rate of the death of $x \in \phi+\delta_{x}$ in configuration $\phi+\delta_{x} \in M(D)$, $x \notin \phi$.

The birth rate functional considered in the present paper is homogeneous in time and space, namely

$$
b\left(\phi, \phi+\delta_{x}\right)=\lambda,
$$

for all $x \in D$ and $\phi \in M(D)$, where $\lambda$ is a positive real number. 
Let $f: \mathbb{R}^{+} \rightarrow \mathbb{R}^{+}$be a non-negative function which we will call the response function of the model. The death rate of the SBD process considered in the present paper is determined by this function through the relation

$$
\mu\left(\phi+\delta_{x}, \phi\right)=\sum_{y \in \phi} f(\|x-y\|)
$$

It is homogeneous in time but not in space: the death rate of $x$ in configuration $\phi+\delta_{x}$ is the shot noise created by $\phi$ at $x$ for the response function $f$.

In the compact domain case, and for bounded birth and death rate functions, the finite time horizon problem can be analyzed by classical Markov chain uniformization techniques and the existence and the uniqueness of the time stationary regimes can be proved using the theory of Markov chains in general state spaces [16].

The object of this paper is the analysis of this type of dynamics in $\mathbb{R}^{d}$. When $D=$ $\mathbb{R}^{d}$, the above Markov approach fails even for the construction of the finite time horizon state. In this case, the birth and death rates (2.1) and (2.2) "describe" a stochastic dynamics, but it is not clear in general whether they uniquely define a stochastic process on the space of counting measures. The main novelty of the present paper is the pathwide construction, based on the random connection model, of a stochastic process having the above birth and death characteristics at any time.

\subsubsection{Death by random connection viewpoint}

Another equivalent description of the dynamics is in terms of a Random Connection Graph (RCG). A RCG [18,7] on a point process $\Phi \in M(D)$ is informally defined as follows: for all $\Phi \in M(D)$, for all unordered pairs $\{x, y\}$ of points of $\Phi$, one samples an independent Bernoulli random variable $Q(x, y)$ with value 1 with probability $c(\|x-y\|)$ and 0 with probability $1-c(\|x-y\|)$. The function $c: \mathbb{R}^{+} \rightarrow[0,1)$ will be referred to as the connection function. The associated random connection model is the random graph on $\phi$ with edges between the points $(x, y)$ such that $Q(x, y)=1$.

Informally, the SBD process studied in this paper can also be obtained by sampling, for all unordered pairs $\{x, y\}$ of points of $\Phi$, an independent exponential random variable $T_{x y}$ with rate $2 f(\|x-y\|)$ and in establishing at this time a lethal connection between $x$ and $y$ which instantly kills either of the two with probability $1 / 2$, independently of everything else. This death can, however, only happen if the points $x$ and $y$ are still alive at time $T_{x y}$, which is not guaranteed as each might have already been killed by other points. It should be clear that, at least in the case where $D$ is compact and the time interval is compact as well, the death rate of any given point $x \in \phi$ is then given by (2.2) as the deaths that occur in state $\phi$ in an infinitesimal interval of time with length $d t$ can be obtained by sampling with probability $1 / 2$ the points connected by edges in a RCG on $\phi$ with connection function $c(r)=2 f(r) d t$. In view of this, it makes sense to call this mechanism death by random connection.

This second view point will be instrumental for constructing the process on $\mathbb{R}^{d}$. 


\subsection{Problem statement}

We will represent the births as a $\mathbb{R}^{d} \times \mathbb{R}$ Poisson point process, $\Psi$, i.e. the births in the time interval $\left(t_{0}, t_{1}\right)$ are $\Psi_{\left(t_{0}, t_{1}\right)}=\Psi_{\mathbb{R}^{d} \times\left(t_{0}, t_{1}\right)}$, a Poisson process on $\mathbb{R}^{d} \times\left(t_{0}, t_{1}\right)$ with intensity measure $\lambda l^{d} \times l\left(t_{0}, t_{1}\right)$, where $l^{d}$ (resp. $l$ ) stands for the Lebesgue measure of $\mathbb{R}^{d}$ (resp. $\mathbb{R}$ ). A point $p \in \Psi$ will also be denoted by $\left(x_{p}, b_{p}\right)$, with $x_{p} \in \mathbb{R}^{d}$ the location of the birth and $b_{p} \in\left(t_{0}, t_{1}\right)$ the time of the birth. The point process $\Psi_{\mathbb{R}^{d} \times\left(t_{0}, \infty\right)}$ will be denoted by $\Psi_{t_{0}}$.

For any two points $p, q \in \Psi$, let $I_{p q}$ and $T_{p q}$ be two random variables independent of everything else with distributions

$$
\begin{aligned}
I_{p q} & =1-I_{q p} \sim \operatorname{Bernoulli}\left(\frac{1}{2}\right), \\
T_{p q} & =T_{q p} \sim\left(b_{p} \vee b_{q}\right)+\operatorname{Exp}\left(2 f\left(\left\|x_{p}-x_{q}\right\|\right)\right) .
\end{aligned}
$$

These quantities have the interpretations alluded to above:

- $T_{p q}$ is the time at which the connection between $p$ and $q$ is realized (it will actually be the death of one of them, if both are alive just before $T_{p q}$ );

- $I_{p q}=1$ if the direction of the connection is from $q$ to $p$ (the dying point will be $p$ if both are alive just before $T_{p q} ; q$ is then said to kill $p$ at time $T_{p q}$ ).

As long as both points are alive, they both "feel" a (time) intensity $f\left(\left\|x_{p}-x_{q}\right\|\right)$ to be killed by the other.

The dynamics of interest can be then captured by the equation:

$$
d_{p}=\inf \left\{T_{p q}: q \in \Psi, d_{q} \geq T_{p q}, I_{p q}=1\right\} .
$$

Note that the condition $d_{q} \geq T_{p q}$ makes this equation recursive.

The general problem can then be stated in the following terms: given some initial condition $\Phi_{0}$, which is some point process in $\mathbb{R}^{d}$, (i) can one construct a solution to (2.3) such that $\Phi_{t}$, the (unordered) set of points alive (born and not yet dead) at time $t$ in this solution, is a point process on $\mathbb{R}^{d}$ with birth and death rates as given in (2.1) and $(2.2)$, for all $t>0$ ? (ii) if so, under what conditions does $\Phi_{t}$ converge in distribution to a limit? (iii) does this limit, when it exists, depend on the initial condition $\Phi_{0}$ ?

For sake of completeness, here is a continuous-time version of the last recursive equation in terms of a stochastic differential equation. Conditionally on $\Psi$, one associates to each pair of points $x, b$ and $y, c$ of $\Psi$, with $x$ and $y$ in $\mathbb{R}^{d}$ and $b$ and $c$ in $\mathbb{R}$, a homogeneous Poisson point processes $N(x, y)$ on $\mathbb{R}$ of intensity $f(\|x-y\|)$. Conditionally on $\Psi$, this countable collection of Poisson point processes is assumed independent. For all bounded sets $C, t \rightarrow \Psi(C, t)$ is a Poisson point process of intensity $\lambda|C|$ and the stochastic differential equation in question is then

$$
\mathrm{d} \Phi_{t}(C)=\Psi(C, \mathrm{~d} t)-\sum_{X \in \Phi_{t}} \sum_{Y \neq X \in \Phi_{t}} \delta_{X}(C) N(X, Y, d t), \quad \forall C .
$$

Note that this equation, which is the usual way of approaching this class of problems, is not essential to our construction. It is only presented here as yet another way of describing the dynamics of interest. 


\subsection{Assumptions on the response function}

Throughout the paper, when $D=\mathbb{R}^{d}$, the following properties on $f$ will be considered:

- Assumption 0: $f$ is non-negative and

$$
\int_{\mathbb{R}^{d} \backslash B(0,1)} f(\|x\|) d x<\infty .
$$

Moreover, we assume $f(0)=0^{3}$.

- Assumption 1:

$$
0<a<\infty \text {, where } a:=\int_{\mathbb{R}^{d}} f(\|x\|) d x .
$$

- Assumption 2: the function $r \rightarrow f(r)$ is monotone non-increasing on $(0, \infty)$.

- Assumption 3: the function $f$ is bounded above. We will then denote by $K$ the upper-bound on $f$.

Assumption 0 is natural in this context; the assumption that $f(0)=0$ makes sense as we always deal with simple point processes. Assumption 1 is used throughout the paper. This assumption is used for proving that events can be sorted out in $\mathbb{R}^{d}$ (Lemma 3.1 and Theorem 3.1). Assumption 2-3 are only needed in the final steps of the construction of the stationary regime; they are not required for the construction on compact sets of time. If Assumption 2 holds (which we do not assume in general), the death rate is higher in regions with many points.

\section{Construction on finite time horizon}

The main question addressed in this section is whether there exists a solution to (2.4) (or equivalently, to (2.3)). We use the connection-death view point described in Sections 2.1.2 and 2.2 to construct these dynamics pathwise over all compact sets of time and space.

It should first be noticed that (2.3) may be problematic if the set

$$
N_{p}=\left\{T_{p q}: q \in \Psi_{t_{0}}\right\}
$$

has accumulation points. The following proposition gives a condition guaranteeing that this is a.s. not the case when $t_{0}$ is finite.

Lemma 3.1. Assume that $t_{0}>-\infty$ and that Assumption 0 holds. Then almost surely none of the sets $N_{p}, p \in \Psi_{t_{0}}$, has accumulation points.

\footnotetext{
${ }^{3}$ The value of $f(0)$ has no influence to process dynamics, but the value $f(0)=0$ can be motivated as the intensity of spontaneous deaths. It is also sometimes a handy way to omit a term containing $f(|X-Y|)$ in the case that $Y=X$.
} 
Proof. For any $p=(x, t) \in \mathbb{R}^{d} \times\left[t_{0}, \infty\right)$, the conditional distribution of $\Psi_{t_{0}}-\delta_{p}$ given that $\Psi_{t_{0}}$ has a point in $p$ is a Poisson process with same distribution as $\Psi_{t_{0}}$ (Slivnyak's theorem, see e.g. [2]). In the following, $\mathbb{E}^{p}$ denotes this conditional expectation, or equivalently, the Palm distribution of $\Psi_{t_{0}}$ at $p$.

To each point $q=(y, s)$ of $\Psi_{t_{0}}$, we associate the point $T_{p q}$ of $\mathbb{R}$.

We show that the intensity measure of this point process on $\mathbb{R}$ is locally finite under the condition given above. For any $u \geq t$,

$$
\begin{aligned}
\mathbb{E}^{p}\left|N_{p} \cap\left(t_{0}, u\right]\right| & =\mathbb{E}^{p} \int_{\mathbb{R}^{d} \times\left(t_{0}, \infty\right)} 1_{T_{p q} \leq u}\left(\Psi_{t_{0}}-\delta_{p}\right)(\mathrm{d} q) \\
& =\mathbb{E} \int_{\mathbb{R}^{d} \times\left(t_{0}, u\right]} 1_{T_{p q} \leq u}\left(\Psi_{t_{0}}\right)(\mathrm{d} q) \\
& =\lambda \int_{\mathbb{R}^{d}} \int_{t_{0}}^{u} \mathbb{P}(\operatorname{Exp}(f(\|x-y\|)) \leq u-(t \vee v)) \mathrm{d} v \mathrm{~d} y \\
& \leq \lambda\left(u-t_{0}\right) \int_{\mathbb{R}^{d}}\left(1-e^{-\left(u-t_{0}\right) f(\|y\|)}\right) \mathrm{d} y \\
& \leq \lambda\left(u-t_{0}\right)\left(\nu_{d}+\left(u-t_{0}\right) \int_{\mathbb{R}^{d} \backslash B(0,1)} f(\|y\|) \mathrm{d} y\right)<\infty
\end{aligned}
$$

where $\nu_{d}$ is the volume of unit ball in $\mathbb{R}^{d}$. Here $\operatorname{Exp}(z)$ denotes an exponential random variable of parameter $z$; the third equality is Campbell's formula; in the last line, we used the inequality $1-e^{-z} \leq z$ and Assumption 0 .

Thus, for all $p$, every finite interval of $[t, \infty)$ contains an a.s. finite number of points of the type $T_{p q}, q \in \Psi_{t_{0}}$. Note that Lemma 3.1 fails with $t_{0}=-\infty$.

\subsection{The Construct Algorithm}

To construct the death process when $t_{0}>-\infty$ and $t_{1}<\infty$, and when the initial condition is empty, we propose below an algorithm that we call the Construct Algorithm.

Within the setting of Section 2.2, the general idea is quite natural: one picks a node, checks its earliest connection (potential death) time; in order to determine whether this is its actual death time, one has to determine whether the death time of the killer is earlier or later than this time (Equation (2.3)); for this, one checks the earliest connection time of the latter, etc.

\section{Algorithm 3.1.}

The Construct Algorithm: Construction of the death process on time interval $\left(t_{0}, t_{1}\right)$.

1 Initialization:

- Every point born in $\left[t_{0}, t_{1}\right]$, say $p=(x, t)$ with $t_{0} \leq t \leq t_{1}$ has a stack of its death sentences. A death sentence for $p$ is a triple $\left(p, q, T_{p q}\right)$, where $q$ is 
a potential killer of $p$, i.e. $I_{p q}=1$. Death sentences are sorted earliest on top $^{4}$

- The algorithm has an investigation stack, initially empty.

2 If the investigation stack is empty, choose, from a pre-defined ordering of all points ${ }^{5}$, the first point whose stack has on top a death sentence with time less than $t_{1}$ and no death certificate, and move the sentence to the investigation stack. If there is no such point, then the procedure ends ${ }^{6}$.

3 Look at the sentence on top of the investigation stack, say $(p, q, T)$, and do one of the following:

- If killer q's stack has on top a death sentence or death certificate with a time larger than $T$, or no death sentence or certificate, then $q$ is alive at $T$ and the execution happens. Change the sentence $(p, q, T)$ into a death certificate with the same data and return it to the top of $p$ 's stack.

- If $q$ has a death certificate earlier than $T$, then the execution is not realized and the sentence $(p, q, T)$ is discarded, i.e. the investigation stack is popped ${ }^{7}$.

- Otherwise move the top sentence of $q$ 's stack to the investigation stack.

4 Go to 2.

To prove that the Construct Algorithm works properly, we start with the following lemma which shows that for all finite intervals $\left(t_{0}, u\right)$ as above, for all predefined ordering of the points, for all sentences, the recursive investigation performed by Construct to determine the status of this sentence ends in finite time.

Lemma 3.2. Assume that $-\infty<t_{0}<t_{1}<\infty$ and that $f$ satisfies Assumptions $0-1$. Then, almost surely, the Construct Algorithm generates no infinite sequence of points $p_{1}, p_{2}, \ldots$ such that the sequence $T_{p_{n} p_{n+1}}$ is non-increasing.

Proof. Notice that since $t_{0}>-\infty$, the sequence $T_{p_{n} p_{n+1}}$ is bounded from below.

The proof uses percolation properties of the Poisson RCG in $\mathbb{R}^{d}[18,7]$. Let us view the arrival locations $x_{p}$ of the points $p$ of $\Psi_{t_{0}}$ arrived until time $u>t_{0}$ as a homogeneous Poisson point process $\Phi$ in $\mathbb{R}^{d}$ with intensity $\lambda\left(u-t_{0}\right)$. The time of arrival $b_{p}$ of point $p$ is seen as an independent mark, uniform on $\left(t_{0}, u\right)$.

Let $J$ be some time interval of $\left(t_{0}, u\right)$. We create an undirected edge (a connection) between the points $x_{p}$ and $x_{q}$ of $\Phi$ if $T_{p q} \in J$. This does not form a RCG because of the marks (in the RCG, one establishes an edge between two points of a Poisson point

\footnotetext{
${ }^{4}$ Note that, under the assumptions of Lemma 3.1, each stack has simple sequential order.

${ }^{5} \mathrm{By}$ this, we mean a bijection between the points of the configuration and $\mathbb{N}$; for instance, points can be sorted in function of their distance to the origin of the Euclidean space, and ties, which may appear with probability zero, can be ordered alphabetically.

${ }^{6}$ We shall see that, under our assumptions of an infinite domain, the algorithm never stops.

${ }^{7}$ That is, the top sentence is deleted and all other sentences are moved up.
} 
process with a probability that depends on their distance only; here the mark of point $x_{p}$ creates a correlation between the edges that connect $x_{p}$ to the other points).

Consider now the model where one creates an undirected edge between $x_{p}$ and $x_{q}$ of $\Phi$ if $M_{x_{p} x_{q}}(J)>0$ where $M_{x_{p} x_{q}}$ is a Poisson point process on $\mathbb{R}$ with intensity $f\left(\left\|x_{p}-x_{q}\right\|\right)$, conditionally independent of everything else given $\left\|x_{p}-x_{q}\right\|$. By a standard coupling argument, this defines a dominating $R C G$, i.e. a RCG where there are more edges than in the original model.

The mean number of connections of point $x \in \Phi$ in this dominating RCG is

$$
\begin{aligned}
\mathbb{E}^{x}\left[\sum_{y \neq x \in \Phi} \mathbb{P}\left(M_{x y}(J)>0 \mid \Psi\right)\right] & \leq \mathbb{E}^{x}\left[\sum_{y \neq x \in \Phi} \mathbb{E}\left(M_{x y}(J) \mid \Psi\right)\right] \\
& =\mathbb{E}^{x}\left[\sum_{y \neq x \in \Phi} l(J) f(\|x-y\|)\right] \\
& =\lambda l(J) \int_{\mathbb{R}^{d}} f(\|z\|) \mathrm{d} z=\lambda l(J) a .
\end{aligned}
$$

Here, $\mathbb{E}^{x}$ refers to the Palm probability of $\Phi$ at $x$; the second bound uses the fact that the probability that a non-negative integer-valued random variable is positive is less than its mean; the last relation follows from Slivnyak's theorem and Campbell's formula. Hence, if the Lebesgue measure $l(J)$ of $J$ is small enough, there is hence no percolation in this dominating RCG $[18,7]$. As a result, there is no percolation in the initial model.

This last property immediately implies that for all $p_{1}$ with $x_{p_{1}}=x$ and all nonincreasing sequences $T_{p_{1}, p_{2}}, T_{p_{2}, p_{3}}, \cdots$ with $T_{p_{1}, p_{2}}=t$, we have $T_{p_{k}, p_{k+1}}<t-\epsilon$ for all $k$ larger than some random but finite $K$. This then proves the result of the lemma by a finite induction.

An important property which remains to be proved is that the result of the Construct algorithm does not depend of the ordering of points that it relies upon. This is the object of the following:

Theorem 3.1. Assume that $t_{0}>-\infty$ and that Assumptions $0-1$ hold. Then almost surely, for every point $p$ born in $\left(t_{0}, t_{1}\right)$, with $t_{1}<\infty$, Construct either determines a unique death time $d_{p} \leq t_{1}$ and the killer, or finds out that $p$ is alive at time $t_{1}$. The result is independent of the order in which the points were enumerated. This uniquely defines the point process $\Phi_{t}$ of nodes alive at time $t$ for all $t>0$.

Proof. Consider Algorithm 3.1. Make Step 2, and consider the set $P$ of all points whose stacks are looked at before the investigation stack is emptied again. By Lemma 3.1, all stacks contain, a.s., only a finite number of sentences with $T_{p q}<t_{1}$, and all times $T_{p q}$ are a.s. distinct. If $P$ is infinite, it contains a sequence with the property appearing in Lemma 3.2. Thus, with probability one, the investigation stack empties. Repeating the cycle, every point, sooner or later and almost surely, either gets a death certificate or has all connection times in its stack larger than $t_{1}$, in which case it is alive at $t_{1}$. 
It remains to show that, almost surely, the resulting configuration does not depend on the pre-defined order in which the points are investigated. Let us consider one realization of the triple $\left(\Psi_{\left(t_{0}, t_{1}\right)},\left\{T_{p q}\right\},\left\{I_{p q}\right\}\right)$ and two different numberings of the points, say $\left\{p^{(1)}\right\}$ and $\left\{p^{(2)}\right\}$. Since every point gets, a.s., a certificate (of death at some time or of survival at time $t_{1}$ ) in both processes, we only need to show that these certificates are a.s. identical in both processes. Assume that for some point $p_{1}$ we have $d_{p_{1}}^{(1)}>d_{p_{1}}^{(2)}$, where the superscripts refer to the two orderings of points. There exists a $p_{2}$ such that $d_{p_{1}}^{(2)}=T_{p_{1} p_{2}}$. Since the sentence $\left(p_{1}, p_{2}, T_{p_{1} p_{2}}\right)$ is present in process 1 and since $p_{1}$ is alive after time $T_{p_{1} p_{2}}$ in process 1 , it must not be killed by $p_{2}$ at $T_{p_{1} p_{2}}$ in process 1 (keeping in mind that $d_{p_{1}}^{(2)}=T_{p_{1} p_{2}}$ implies $I_{p_{1} p_{2}}=1$ ). Hence, it must be that $p_{2}$ is already dead at that time, i.e.

$$
d_{p_{2}}^{(1)}<T_{p_{1} p_{2}}=d_{p_{1}}^{(2)}
$$

Consider now process 2 . The fact that $d_{p_{1}}^{(2)}=T_{p_{1} p_{2}}$ implies that

$$
T_{p_{1} p_{2}}=d_{p_{1}}^{(2)}<d_{p_{2}}^{(2)}
$$

Hence, in view of $d_{p_{2}}^{(1)}<d_{p_{1}}^{(2)}$, we have $d_{p_{2}}^{(2)}>d_{p_{2}}^{(1)}$. Now, this reasoning can be continued, leading to an infinite sequence of distinct points $p_{n}$ such that

$$
d_{p_{1}}^{(1)}>d_{p_{1}}^{(2)}>d_{p_{2}}^{(1)}>d_{p_{3}}^{(2)}>d_{p_{4}}^{(1)}>\cdots
$$

and within this sequence ( $i$ alternating between 1 and 2) $d_{p_{n}}^{(i)}=T_{p_{n} p_{n+1}}$ for $n=1,2, \ldots$. But this sequence is exactly of the kind whose existence is a.s. denied by Lemma 3.2.

Below we will take $t_{0}=0$. The Construct Algorithm can be seen as a measurable mapping from $\left(M\left(\mathbb{R}^{d} \times \mathbb{R}\right),(0, \infty)^{\mathbb{N}},\{0,1\}^{\mathbb{N}}\right)$ to $M\left(\mathbb{R}^{d} \times \mathbb{R}\right)$ with

$$
\operatorname{Construct}\left(\Psi_{\left(0, t_{1}\right)},\left\{T_{p q}\right\},\left\{I_{p q}\right\}\right):=\left\{x_{p}, d_{p}\right\}_{p \in \Psi_{\left(0, t_{1}\right)}},
$$

where, in the case $t_{1}<\infty$, we set $d_{p}=\infty$ for points living at time $t_{1}$.

\subsection{More general initial conditions}

The initial condition of the Construct algorithm was empty at time $t_{0}$ since the stacks were defined from the arrivals in $\left(t_{0}, t_{1}\right)$. It will be useful below to extend this to an initial condition made of a point process $\mathcal{Z}_{0}$ of nodes already present (i.e. born) at time $t_{0}$ and having independent pairwise random connections and killing direction variables as those defined above. In this case, an initial stack is built for each node of $\mathcal{Z}_{0} \cup \Psi_{\left(t_{0}, t_{1}\right)}$, containing its sorted sentences. If the point process $\mathcal{Z}_{0}$ satisfies the property in Lemmas 3.1 and 3.2 namely if

1. for every $z$ in $\mathcal{Z}_{0} \cup \Psi_{\left(t_{0}, t_{1}\right)}$, the set of all $T_{z, w}, w \in \mathcal{Z}_{0} \cup \Psi_{\left(t_{0}, t_{1}\right)}$ which belong to $\left(t_{0}, t_{1}\right)$ is finite for $-\infty<t_{0}<t<\infty$; 
2. there is no infinite sequence of points $z_{1}, z_{2}, \ldots$ of $\mathcal{Z}_{0} \cup \Psi_{\left(t_{0}, t_{1}\right)}$ such that the sequence $T_{z_{n} z_{n+1}}$ is non-increasing,

then there is no difficulty in running Construct on this initial condition. The first assumption is used to guarantee that there will be a finite number of sentences in the stack of any point. The second one is used to show that the construction returns a death time or a certificate of survival at $t_{1}$ to every point of $\mathcal{Z}_{0} \cup \Psi_{\left(t_{0}, t_{1}\right)}$. The arguments are exactly the same.

Throughout the rest of the paper, the initial condition will be assumed to be a motion invariant point process [2] satisfying the conditions 1 and 2 given above.

Here are a few examples where this condition is satisfied. If $\mathcal{Z}_{0}$ is Poisson, homogeneous and independent of $\Psi_{\left(t_{0}, t_{1}\right)}$, this follows from Lemma 3.2. By a direct monotonicity argument, the same holds if $\mathcal{Z}_{0}$ is any compatible thinning ${ }^{8}$ of an independent homogeneous Poisson point process. This compatible thinning can be based on the independent pairwise connections and killing directions. In particular let $\Phi_{t_{1}}$ denote the point process, built by Construct, of nodes living at time $t_{1}$ when the system starts empty at time $t_{0}$. This is a motion invariant thinning of $\Psi_{\left(t_{0}, t_{1}\right)}$ based on these pairwise variables. One can hence apply Construct on $\left[t_{1}, t_{2}\right)$ to the initial condition $\mathcal{Z}_{0}=\Phi_{t_{1}}$ for all $t_{1}<t_{2}<\infty$.

\subsection{The double card version of Construct}

The Construct Algorithm could also be defined as follows: in the initialization, for each connection time $T_{p q}$, put a sentence $\left(p, q, T_{p q}\right)$ in $p$ 's stack and a sentence $\left(q, p, T_{p q}\right)$ in $q$ 's stack (remember that $T_{p q}=T_{q p}$ ). The values of the $I_{p q}$ 's are not drawn beforehand, so that we speak duel times rather than of death sentences. In Step 2 of Construct, copy (instead of move) the top sentence of the next point whose top sentence carries a time less than $t_{1}$ and is not a death certificate to the investigation stack. In step 3 , there are three alternatives: (i) if $q$ 's stack has a death certificate on top, then $p$ 's stack and the investigation stack are popped; (ii) if $q$ 's top sentence carries a duel time less than $T_{p q}$, that sentence is copied to the investigation stack; (iii) in the remaining case, $q$ 's top sentence is $\left(q, p, T_{q p}\right)$; now the $I_{p q}$ variable is drawn, the loser's top sentence is replaced by a death certificate, and the killer's stack and the investigation stack are popped. It is obvious that this variant, which will be referred to as the double card version of the algorithm, performs similarly to the initial one (although the investigation order is not exactly the same).

The double card version makes it clear that the direction of the interaction (i.e. the value of $I_{p q}$ ) need not be specified before the step when it is really needed in the algorithm at the realization of a duel. Another nice feature is that full information on the $\left\{T_{p q}\right\}$ sequence remains in the stacks. Indeed, for an unrealized duel, which can only happen when either or both duelists are dead before the execution time, a copy of the sentence remains in the stack of at least one of the two duelists.

\footnotetext{
${ }^{8}$ By compatible thinning, we mean a thinning where the retention decisions are marks of the point process.
} 


\section{Initial condition and coupling}

In this section, we investigate how additions to the initial condition perturb the history of all other points. This is done through a coupling, called Construct $^{Z}$, which allows one to jointly build the histories with and without these additions. This construction is subtle because of the non-monotonicity of the dynamics and the fact that point processes with infinitely many points have to be considered for such perturbations. The main result is a quantification of the speed at which the effect of the initial condition fades away in any compact. The finiteness of the effect of this perturbation will be instrumental to construct the steady state through a coupling from the past in Section 7 .

\subsection{Augmenting the initial condition}

Below, we consider two systems: (1) that with an empty set of nodes as initial condition (as in Construct); (2) that with an initial condition consisting of a point process $\mathcal{Z}_{0}$ in $\mathbb{R}^{d} \times\{0\}$, satisfying the conditions of Subsection 3.2 and representing some additional set of points born at time 0 . The point process $\mathcal{Z}_{0}$ will be called the augmentation point process. The first case is a special case of the second one (with $\mathcal{Z}_{0}=\emptyset$ ) and will be referred to as the non-augmented case.

Our aim below will be to jointly build two parallel executions of the killing history: that with this augmentation and that without. The coupling consists of using the same sequences of connections $\left(\left\{T_{p q}\right\}\right)$ for common points (the points of $\left.\Psi_{\left(0, t_{1}\right)}\right)$. The addition of $\mathcal{Z}_{0}$ has non-monotonic effects on the life times of common points, with some points having their lifetime extended and others shortened. In the algorithm described below, at any given time, we call $Z$ points the points that are alive in the augmented process and are dead in the non augmented process (i.e. with a death time already determined in the non-augmented process and not yet determined in the augmented one $)^{9}$. Conversely we will call $A$ points the points that are dead in the augmented process and alive in the original process (i.e. with a death time already determined in the augmented process and not yet in the non-augmented one). ${ }^{10}$.

At any given time, $\mathrm{Z}$ points and A points will be called special points and the other points will be called regular. The basic principles of the joint execution are as follows:

- The killing of a regular point by another regular point determines the death times of the former in the two processes (these death times are equal).

- If a $\mathrm{Z}$ point kills a regular point, this determines the death time of the latter in the augmented process. This regular point becomes an A point and is kept in the algorithm until its death time is determined in the non-augmented process.

\footnotetext{
${ }^{9}$ It makes sense to call the points of $\mathcal{Z}_{0} \mathrm{Z}$ points as well.

${ }^{10}$ In more mnemonic vocabulary, the $\mathrm{Z}$ points could be called "zombies", because they are simultaneously dead and alive, and the A points "anti-zombies".
} 

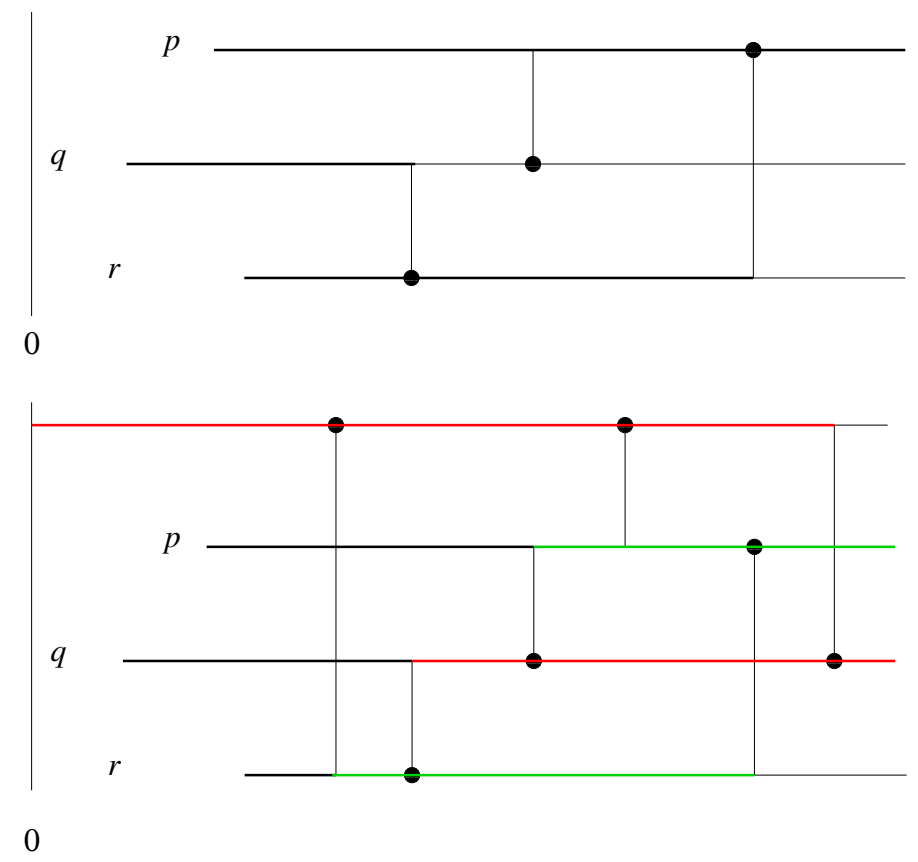

Figure 1: Two coupled killing processes. Time is on the $x$ axis; space on the $y$ axis. Dots associated with vertical arrows indicate killers. Upper figure: a killing process with three points. Lower figure: same process with an added point born at time zero. Colors: black: regular point; red: Z point; green: A point.

- If an A point kills a regular point, this determines the death time of the latter in the non-augmented process. This point becomes a $\mathrm{Z}$ point and is kept in the algorithm until its death time is determined in the augmented process.

- If a regular point kills a $\mathrm{Z}$ point, this determines the death time of the latter in the augmented process, and this $\mathrm{Z}$ point can be forgotten as its two death times are now determined in both processes.

- If a regular point kills an A point, this determines the death time of the latter in the non-augmented process, and the A point can be forgotten for the same reasons as above.

- If a $\mathrm{Z}$ point (resp. an $\mathrm{A}$ point) kills another $\mathrm{Z}$ point (resp. A point), this determines the death time of the latter in the augmented (resp. non-augmented) process and the killed point can be forgotten.

- Z points and A points cannot kill each other as they belong to different processes.

See Figure 1 for an illustration. 
The Construct ${ }^{Z}$ Algorithm described below generates the announced coupling of the original and the augmented process. It simultaneously builds two sequences $\left\{e_{p}\right\}_{p \in \Psi_{\left(0, t_{1}\right)}}$ and $\left\{e_{p}^{\prime}\right\}_{p \in \mathcal{Z}_{0} \cup \Psi_{\left(0, t_{1}\right)}}$ that will be shown later to coincide with the death sequences of the non-augmented and the augmented systems, respectively. It does so by maintaining the list of $\mathrm{Z}$ points and $\mathrm{A}$ points at all times.

Algorithm 4.1. Construct ${ }^{Z}$ ("Construct with Z points").

Input: $\mathcal{Z}_{0}, \Psi_{\left(0, t_{1}\right)},\left\{T_{p q}\right\}_{p, q \in \mathcal{Z}_{0} \cup \Psi_{\left(0, t_{1}\right)}},\left\{I_{p q}\right\}_{p, q \in \mathcal{Z}_{0} \cup \Psi_{\left(0, t_{1}\right)}}$.

Output: $\left\{e_{p} \in\left[0, t_{1}\right]\right\}_{p \in \cup \Psi_{\left(0, t_{1}\right)}},\left\{e_{p}^{\prime} \in\left[0, t_{1}\right]\right\}_{p \in \mathcal{Z}_{0} \cup \Psi_{\left(0, t_{1}\right)}}$.

The algorithm has an investigation stack (IS), initially empty.

1. Initialization:

- For each $p \in \mathcal{Z}_{0} \cup \Psi_{\left(0, t_{1}\right)}$, build a stack $S_{p}$ of sentence of the form $\left(p, q, T_{p q}\right)$, where the $T_{p q}$ variables are sorted in increasing order (earliest time on top) ${ }^{11}$;

- For all $p, e_{p}:=e_{p}^{\prime}:=\infty$;

- For all $z \in \mathcal{Z}_{0}$, one maintains the point sets $\mathcal{A}(z)$ ("A points") and $\mathcal{Z}(z)$ ("Z points") offspring of $z$; initially, $\mathcal{A}(z):=\emptyset$ and $\mathcal{Z}(z):=\{z\}$; if at some time $p \in \mathcal{Z}(z)$ or $p \in \mathcal{A}(z)$, we define $\mathfrak{z}(p)=z$ (the value will be uniquely defined); denote (at all times) $\mathcal{A}=\cup_{z \in \mathcal{Z}_{0}} \mathcal{A}(z), \mathcal{Z}=\cup_{z \in \mathcal{Z}_{0}} \mathcal{Z}(z)$; points that bear neither label are called "regular points" and denoted by $\mathcal{R}$;

- Call a point $p$ finished, if either $p \in \mathcal{Z}_{0}$ and $e_{p}^{\prime}<\infty$, or $p \in \Psi_{\left(0, t_{1}\right)}$ and $e_{p} \vee e_{p}^{\prime}<\infty$, or $S_{p}$ 's top sentence has $T_{p q} \geq t_{1}$.

2. If IS is empty, choose, from a pre-defined ordering of all points, the first unfinished point $p$ such that the top sentence of $S_{p}$ has $T_{p q}<t_{1}$, and copy this sentence to IS.

3. Look at the top sentence of IS, say $\left(p, q, T_{p q}\right)$.

- if $q$ is finished, pop both $S_{p}$ and IS; go to Step 2;

- if $S_{q}$ 's top sentence has $T_{q r}<T_{p q}$, copy this last sentence to IS; go to Step 3 .

- Otherwise, $\left(q, p, T_{q p}\right)$ is on the top of $S_{q}$; proceed to next step.

4. Do one of the following (if the appropriate case is missing, interchange $p$ and $q$ ): $p, q \in \mathcal{R}:$

- if $I_{p q}=1, e_{p}:=e_{p}^{\prime}:=T_{p q} ;$ pop $S_{q}$;

- if $I_{p q}=0, e_{q}:=e_{q}^{\prime}:=T_{p q} ;$ pop $S_{p}$;

$p \in \mathcal{Z}$ and $q \in \mathcal{R}$ :

\footnotetext{
${ }^{11}$ We use here the double card version so that for all sentences of the form $\left(p, q, T_{p q}\right)$ stored in $p$ 's stack, a sentence with the same data is also stored in $q$ 's stack.
} 
- if $I_{p q}=1, e_{p}^{\prime}:=T_{p q} ; \mathcal{Z}(\mathfrak{z}(p)):=\mathcal{Z}(\mathfrak{z}(p)) \backslash\{p\} ;$ pop $S_{q}$;

- if $I_{p q}=0, e_{q}^{\prime}:=T_{p q} ; \mathcal{A}(\mathfrak{z}(p)):=\mathcal{A}(\mathfrak{z}(p)) \cup\{q\} ;$ pop $S_{p}$ and $S_{q}$;

$p \in \mathcal{A}$ and $q \in \mathcal{R}$ :

- if $I_{p q}=1, e_{p}:=T_{p q} ; \mathcal{A}(\mathfrak{z}(p)):=\mathcal{A}(\mathfrak{z}(p)) \backslash\{p\} ;$ pop $S_{q}$;

- if $I_{p q}=0, e_{q}:=T_{p q} ; \mathcal{Z}(\mathfrak{z}(p)):=\mathcal{Z}(\mathfrak{z}(p)) \cup\{q\} ;$ pop $S_{p}$ and $S_{q}$;

$p, q \in \mathcal{Z}:$

- if $I_{p q}=1, e_{p}^{\prime}:=T_{p q} ; \mathcal{Z}(\mathfrak{z}(p)):=\mathcal{Z}(\mathfrak{z}(p)) \backslash\{p\} ;$ pop $S_{q}$;

- if $I_{p q}=0, e_{q}^{\prime}:=T_{p q} ; \mathcal{Z}(\mathfrak{z}(q)):=\mathcal{Z}(\mathfrak{z}(q)) \backslash\{q\} ;$ pop $S_{p}$;

$p, q \in \mathcal{A}:$

- if $I_{p q}=1, e_{p}:=T_{p q} ; \mathcal{A}(\mathfrak{z}(p)):=\mathcal{A}(\mathfrak{z}(p)) \backslash\{p\} ;$ pop $S_{q}$;

- if $I_{p q}=0, e_{q}:=T_{p q} ; \mathcal{A}(\mathfrak{z}(q)):=\mathcal{A}(\mathfrak{z}(q)) \backslash\{q\} ;$ pop $S_{p}$;

$p \in \mathcal{Z}$ and $q \in \mathcal{A}$ :

- pop $S_{p}$ and $S_{q}$.

5. Pop IS and go to Step 2.

Remark 4.1. If the set $\mathcal{Z}_{0}$ is empty, Construct ${ }^{Z}$ reduces to the double card version of Construct (see Subsection 3.3); in this case, the first case in Step 4 is always met.

Remark 4.2. The algorithm visits Step 4 after a finite number of steps (and hence infinitely often) because each of the investigations completes in a finite number of steps.

Remark 4.3. In the second bullet of cases 2 and 3 in Step 4, one discards the top sentences of both $p$ and $q$ because $q$ does not kill $p$ but only labels it, and the connection between the two can be forgotten.

\subsection{Properties of the Construct ${ }^{Z}$ map}

Let us now see in detail what Construct $^{Z}$ does. Let

$$
\left\{x_{p}, d_{p}\right\}_{p \in \Psi_{\left(0, t_{1}\right)}}=\operatorname{Construct}\left(\Psi_{\left(0, t_{1}\right)},\left\{T_{p q}\right\},\left\{I_{p q}\right\}\right),
$$

where $\left\{T_{p q}\right\}$ and $\left\{I_{p q}\right\}$ are indexed by $p, q \in \Psi_{\left(0, t_{1}\right)}$ and let

$$
\left\{x, p, d_{p}^{\prime}\right\}_{p \in \mathcal{Z}_{0} \cup \Psi_{\left(0, t_{1}\right)}}=\operatorname{Construct}\left(\mathcal{Z}_{0} \cup \Psi_{\left(0, t_{1}\right)},\left\{T_{p q}\right\},\left\{I_{p q}\right\}\right),
$$

where $\left\{T_{p q}\right\}$ and $\left\{I_{p q}\right\}$ are now indexed by $p, q \in \mathcal{Z}_{0} \cup \Psi_{\left(0, t_{1}\right)}$. In these last definitions, we use the same sequences $\left\{T_{p q}\right\}$ and $\left\{I_{p q}\right\}$ as in Construct $^{Z}$. Then we have:

Theorem 4.1. Under Assumptions 0-1, the following claims hold almost surely:

1. The algorithm Construct ${ }^{Z}$ runs unambiguously and every point gets finished (in the sense defined at the end of the initialization) in finite time. For all $p \in \Psi_{\left(0, t_{1}\right)}$, $e_{p}=d_{p}$, whereas for all $\left.p \in \mathcal{Z}_{0} \cup \Psi_{\left(0, t_{1}\right)}, d_{p}^{\prime}=e_{p}^{\prime}\right)$. 
2. For each $z \in \mathcal{Z}_{0}$, the Construct ${ }^{Z}$ algorithm (implicitly) generates the set-valued stochastic processes $\left(A_{t}(z)\right)_{t \in\left[0, t_{1}\right)}$ and $\left(Z_{t}(z)\right)_{t \in\left[0, t_{1}\right)}$ representing, respectively, the $A$ points and $Z$ points originating from $z$ and living at time $t$. These sets satisfy the conditions

$$
\begin{array}{ll}
p \in A_{t}(z) \text { for some } z & \Leftrightarrow \quad d_{p}^{\prime} \leq t<d_{p}, \\
p \in Z_{t}(z) \text { for some } z & \Leftrightarrow \quad d_{p} \leq t<d_{p}^{\prime} .
\end{array}
$$

The "families" of offsprings

$$
O_{t_{1}}(z)=\bigcup_{t \in\left[0, t_{1}\right)} A_{t}(z) \cup \bigcup_{t \in\left[0, t_{1}\right)} Z_{t}(z)
$$

of distinct z's are disjoint.

Proof. First note that if, in any phase of the algorithm, a point $p$ belongs to $\mathcal{A}(z) \cup \mathcal{Z}(z)$ for some $z \in \mathcal{Z}_{0}$, then $z$ is unique and we can thus denote it as $\mathfrak{z}(p)$. Indeed, this holds true for the initial situation where $\mathcal{A}(z) \cup \mathcal{Z}(z)=\{z\}$, and any given point can be added to some $\mathcal{A}(z) \cup \mathcal{Z}(z)$ only once. Thus, the steps of the algorithm are unambiguously defined.

Second, note that we now use the double card version as discussed in Subsection 3.3. All points get finished (a.s.) by the argumentation used for proving the same for Construct.

Since the algorithm clearly fixes the times when a point becomes or ceases to be a $\mathrm{Z}$ point or A point, it is obvious that the processes $\left(A_{t}(z)\right)_{t \in\left[0, t_{1}\right)}$ and $\left(Z_{t}(z)\right)_{t \in\left[0, t_{1}\right)}$ are well defined.

A further examination of the algorithm yields the conditions (4.1). We also see why $\mathrm{Z}$ points and A points don't interact (last case of step 4): if $p \in A_{t}(z)$ and $q \in Z_{t}\left(z^{\prime}\right)$, at time $t, p$ is dead in the augmented scenario and $q$ is dead in the original scenario. Claim 2 is now proven, since the last subclaim just states the uniqueness discussed already in the beginning of the proof.

To show that $d_{p}=e_{p}$ for all $p$, note that $\mathrm{Z}$ points are points that already have the $e$-value set. When a $\mathrm{Z}$ point kills a regular point, the latter receives an $e^{\prime}$-value and becomes an A point, but this has no effect on the setting of subsequent $e_{p}$-values. If $\mathrm{Z}$ points were considered as finished (that is, dead), the $e_{p}$-values would be the same.

This is illustrated in Figure 2.

Similarly, the A points have no effect on the setting of $e_{p}$-values - if we are interested only in the latter, A points could as well be considered as finished. This completes the proof of Claim 1.

Theorem 4.2. Under Assumptions $0-1$, the families $O_{t_{1}}(z)$ are finite; this also holds in the case $t_{1}=\infty$.

Proof. Clearly it suffices to consider the case $t_{1}=\infty$. Define the filtration $\mathbb{F}=\left(\mathcal{F}_{t}\right)_{t \geq 0}$ as

$$
\mathcal{F}_{t}=\sigma\left(\mathcal{Z}_{0} \cup \Psi_{(0, t)}\right) \vee \sigma\left(\left(T_{x y}, I_{x y}\right): x, y \in \mathcal{Z}_{0} \cup \Psi_{(0, t]} \text { s.t. } T_{x y} \leq t\right)
$$




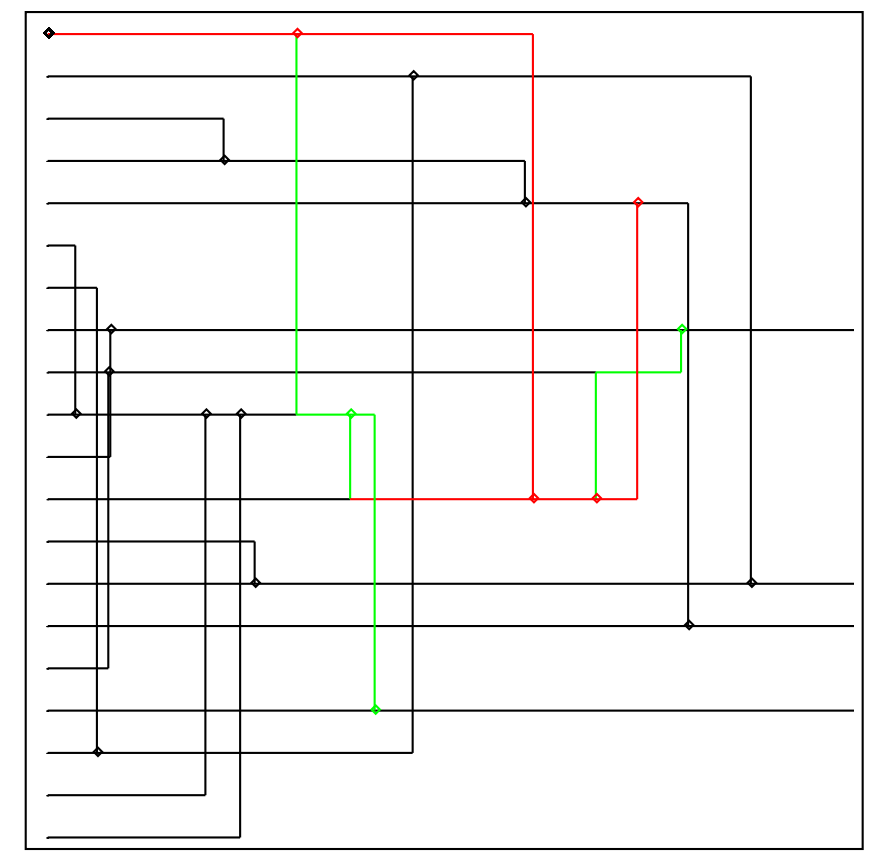

Figure 2: Time is on the $x$ axis; space on the $y$ axis. All nodes are born at the same time to increase readability. Dots indicate killers. Compared to Figure 1, only relevant vertical edges are represented. Colors: black: regular point; red: Z point; green: A point. Black plus green define the $e$ variables. Black plus red define the $e^{\prime}$ variables.

Obviously, if $x, y \in \mathcal{Z}_{0} \cup \Psi_{(0, \infty)}$, then $T_{x y}$ is an $\mathbb{F}$-stopping time. Further, $I_{x y}$ is not

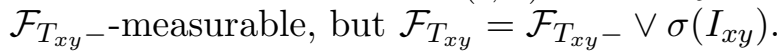

Let us fix a point $z \in \mathcal{Z}_{0}$ and let $S_{t}(z)=A_{t}(z) \cup Z_{t}(z)$. For $t \geq 0$, define the 'set of relevant points' as

$$
U_{t}(z)=\left\{x \in \mathcal{Z}_{0} \cup \Psi_{(0, \infty)}: \exists y \in S_{t}(z): T_{x y}>t\right\} .
$$

We define inductively a non-decreasing sequence of stopping times $T_{n}$ and a sequence of random variables $J_{n} \in\{-1,0,1\}$ by setting $T_{0}=J_{0}=0$ and

$$
T_{n+1}= \begin{cases}\infty, & \text { if } T_{n}=\infty, \\ \inf \left\{T_{x y}: x \in U_{T_{n}}, y \in S_{T_{n}}(z)\right\}, & \text { otherwise, }\end{cases}
$$

with inf $\emptyset=\infty$. Assume that, for some $n, S_{T_{n}}$ is finite and non-empty. By Lemma 3.1 we have, a.s., $T_{n}<T_{n+1}<\infty$, and $S_{t}$ does not change on $\left[T_{n}, T_{n+1}\right)$. Let now $T_{n+1}=T_{x y}$, where $y \in S_{T_{n}}(z)$, and if $x \in S_{T_{n}}(z)$, we choose for definiteness $x$ as being farther from the origin than $y$. At time $T_{n+1}$, one of the following takes place:

- Case 0: $x$ is finished before time $T_{x y}$, or $x$ is, before $T_{n+1}$, a special point of the kind opposite to that of $y$; then $S_{T_{n+1}}(z)=S_{T_{n}}(z)$. 
- Case 1: $x$ is a regular point. Then the chances, determined by $I_{x y}$, are $\frac{1}{2}$ that $S_{T_{n+1}}(z)=S_{T_{n}}(z) \cup\{x\}$ (with $x$ being killed by a Z point or an A point and being transformed to the opposite kind) and $\frac{1}{2}$ that $S_{T_{n+1}}(z)=S_{T_{n}}(z) \backslash\{y\}(y$ being killed by $x$ ).

- Case 2: $x$ is a special point of the same kind as $y$, and $x \notin S_{T_{n}}(z)$. Now the chances are $\frac{1}{2}, \frac{1}{2}$ that $S_{T_{n+1}}(z)=S_{T_{n}}(z)$ or $S_{T_{n+1}}(z)=S_{T_{n}}(z) \backslash\{y\}$.

- Case 3: $x$ and $y$ are special points of same kind, and $x \in S_{T_{n}}(z)$. Then $S_{T_{n+1}}(z)=$ $S_{T_{n}}(z) \backslash\{x\}$ or $S_{T_{n+1}}(z)=S_{T_{n}}(z) \backslash\{y\}$, depending on $I_{x y}$.

In Case 0 we set $J_{n+1}=0$, and in the remaining cases $J_{n+1}=-1+2 I_{x y}$. On the other hand, if $T_{n+1}=\infty$, we set $J_{n+1}=0$, and interpret $S_{T_{n+1}}(z)=S_{T_{n}}(z)$. Note that $S_{T_{n+1}}$ is finite in every case, and

$$
s_{n}:=\left|S_{T_{n}}(z)\right| \leq 1+\sum_{k=1}^{n} J_{k}, \quad n \geq 0 .
$$

In fact, the sequence $s_{n}$ is an $\left(\mathcal{F}_{T_{n}}\right)$-supermartingale. If $T_{n}=\infty$ for some $n$, we clearly have $\left|O_{\infty}(z)\right|<\infty$. If $T_{n}<\infty$ for all $n$ but $J_{n} \neq 0$ for only finitely many $n$, then $O_{T_{n}}$ remains unchanged for $n \geq n_{0}$, and $T_{n} \rightarrow \infty$ by Lemma 3.1, thus again $\left|O_{\infty}(z)\right|<\infty$. Finally, assume that we have with positive probability $T_{n}<\infty$ for all $n$ and $J_{n} \neq 0$ for infinitely many $n$. Now, such $J_{n}$ s are independent random variables taking values \pm 1 with probabilities $\frac{1}{2}, \frac{1}{2}$. Since a symmetric random walk on $\mathbb{N}$ hits zero with probability 1 , there is a finite random number $c$ such that $1+\sum_{k=1}^{c} J_{k}=0$. Now, by $(4.2), s_{c}=0$, and we get $T_{c+1}=\infty$, which contradicts the assumption. This concludes the proof.

\subsection{Section summary}

Let us summarize this section by focusing on the case where the augmentation point process $\mathcal{Z}_{0}$ is translation invariant in $\mathbb{R}^{d}$. We established the following results:

1. Theorem 4.1 uniquely defines the marked point process $\widetilde{\Phi}_{t}$ of nodes which are not finished at time $t<\infty$ under Construct $^{Z}$; the marks belong to the set $\{\mathcal{R}, \mathcal{A}, \mathcal{Z}\}$. The points with mark $\mathcal{R}$ are regular points, which are alive both in the augmented and the non-augmented processes, whereas those with mark $\mathcal{A}$ (resp. $\mathcal{Z}$ ) are $\mathrm{A}$ points (resp. Z points) with a life shorter (resp. longer) in the augmented process compared to the non-augmented one.

2. Theorem 4.1 also shows that the points of $\widetilde{\Phi}_{t}$ with marks in $\mathcal{R} \cup \mathcal{Z}$ form a stationary point process $\Phi_{t}^{\prime}$ which coincides with that built by Construct at time $t$ when the initial condition is $\mathcal{Z}_{0}$. Similarly, the points of $\widetilde{\Phi}_{t}$ with marks in $\mathcal{R} \cup \mathcal{A}$ form a stationary point process $\Phi_{t}$ which coincides with that built by Construct at time $t$ when the initial condition is $\emptyset$.

3. Theorem 4.2 shows that the set of special points of $\left(\widetilde{\Phi}_{t}\right)_{t \geq 0}$ which are offsprings of a given point $z \in \mathcal{Z}_{0}$ has a finite cardinality a.s. The distribution of this collection of sets is translation invariant. 


\section{Non-degeneracy of transient densities}

This section is still focussed on the effect of a change in the initial condition. It is based on the construction of the last section and complements it by an analytical machinery based on densities. The machinery in question will lead to differential equations for densities and moment measures in Sections 6 and 8, and will be instrumental in the coupling from the past. This section makes the first steps in this direction by proving that the densities in question exist and are non degenerate.

From now on, the augmentation point process $\mathcal{Z}_{0}$ used for the initial condition is assumed to be motion invariant in $\mathbb{R}^{d}$ and to satisfy the assumptions of Subsection 3.2.

\section{$5.1 \quad$ Tightness}

We first give a simple stochastic comparison argument showing that the stochastic processes built by Construct are tight, which in turn implies that densities admit a uniform upper bound.

Let us define an elementary mutual service process with parameters $(\tilde{\lambda}, \tilde{\mu})$ as the birth-death process whose birth and death intensities in state $j$ are

$$
\lambda_{j} \equiv \tilde{\lambda}, \quad \mu_{j}=j(j-1) \tilde{\mu}, \quad j=0,1,2 \ldots
$$

Note that although an elementary mutual service process may start from state 0 , it cannot reach 0 from any other state $j>0$.

Proposition 5.1. Under Assumptions $0-2$, if $\mathcal{Z}_{0}$ is a translation invariant thinning of a Poisson process on $\mathbb{R}^{d}$, then the process $\left(\Phi_{t}^{\prime}\right)_{t \geq 0}$ built by Construct satisfies the following properties:

1. For any $t>0, \Phi_{t}^{\prime}$ is spatially stationary and ergodic.

2. Let $b>0$ be sufficiently small to satisfy $f(b \sqrt{d})>0$; tessellate $\mathbb{R}^{d}$ into cubes $C_{i}$ of side $b$ indexed by $i \in \mathbb{Z}^{d}$, assuming that the center of $C_{0}$ is the origin. Then there exists an elementary mutual service process $U(i)$ with parameters $\tilde{\lambda}=\lambda b^{d}$, $\tilde{\mu}=2 f(b \sqrt{d})$, such that, a.s.,

$$
\Phi_{t}^{\prime}\left(C_{i}\right) \leq U_{t}(i), \quad t \geq 0, i \in \mathbb{Z}^{d}
$$

and the processes $U(i)$ are independent given their initial states

$$
U_{0}(i)=\mathcal{Z}_{0}\left(C_{i}\right), \quad i \in \mathbb{Z}^{d} .
$$

3. The intensities $\beta_{\Phi_{t}^{\prime}}$ satisfy the bound

$$
\beta_{\Phi_{t}^{\prime}}<c, \quad t \geq 0
$$

with $c$ a finite constant. 
4. For all positive integers $k$ and for all bounded Borel sets $C, \mathbb{E}\left(\Phi_{t}^{\prime}(C)^{k}\right)$ is uniformly bounded in $t$.

Proof. Claim 1: the (space) stationarity and the ergodicity follow from the fact that the point process $\Phi_{t}^{\prime}$ is a translation invariant thinning of an independently marked stationary and ergodic point process.

Claim 2: by a classical coupling argument, we can construct the processes $U_{t}(i)$ on an extension of the probability space of $\left(\Phi_{t}^{\prime}\right)$ so that $(5.1)$ and the conditional independence hold. Set the initial states of $U_{t}(i)$ according to (5.2). For each $i \in \mathbb{Z}^{d}$, we can obviously make the up-jumps of $\Phi^{\prime}\left(C_{i}\right)$ and $U_{t}(i)$ identical. For down-jumps, assume that $\Phi_{t}^{\prime} \cap C_{i}=\left\{X_{1}, \ldots, X_{m}\right\}$ with $m \geq 2$, and that $U_{t}(i)=j \geq m$. Given $\Phi_{t}^{\prime}$, the times $T_{X_{i_{1}} X_{i_{2}}}$ are independent exponentially distributed random variables with parameters $2 f\left(\left\|X_{i_{1}}-X_{i_{2}}\right\|\right) \geq \tilde{\mu}$, respectively. If $j=m$,

$$
\min _{i_{1}} d_{X_{i_{1}}} \leq \min _{i_{1}, i_{2}} T_{X_{i_{1}} X_{i_{2}}} \stackrel{\text { (st) }}{\leq} \operatorname{Exp}(j(j-1) \tilde{\mu}),
$$

with st denoting stochastic ordering. The claim then follows from these observations.

Claims 3 and 4 follow from Claim 2 since, except for state 0 , an elementary mutual service process is dominated by an $\mathrm{M} / \mathrm{M} / \infty$ queue with the same parameters, whose stationary distribution is Poisson.

Remark 5.1. For all thinned Poisson initial conditions $\mathcal{Z}_{0}$ (such initial conditions satisfy the above assumptions), one can adapt the last proof and obtain analogues of Proposition 5.1 for

1. The point process $\Phi_{t}^{\prime}=\Phi_{t, \mathcal{Z}_{0}}$ built by Construct;

2. The point process $\widetilde{\Phi}_{t}$ built by Construct ${ }^{Z}$; for showing this last property, one can use the fact that $\widetilde{\Phi}_{t}$ is bounded from above by the superposition of the point processes $\Phi_{t}$ and $\Phi_{t}^{\prime}$, which both satisfy the desired properties.

Lemma 5.1. Let $\mathbb{E}_{\chi}^{0}$ stand for the Palm probability of a point process $\chi$. Under Assumptions $0-3$, there exists a finite constant $c$ such that

$$
\beta_{\Phi_{t}} \mathbb{E}_{\Phi_{t}}^{0} \sum_{X \in \Phi_{t}} f(\|X\|) \leq c, \quad \forall t \in \mathbb{R}
$$

More generally, for all positive integers $k$, there exists a finite constant $c_{k}$ such that

$$
\beta_{\Phi_{t}} \mathbb{E}_{\Phi_{t}}^{0}\left[\left(\sum_{X \in \Phi_{t}} f(\|X\|)\right)^{k}\right] \leq c_{k}, \quad \forall t \in \mathbb{R} .
$$

Proof. Using the product form upper bound of Proposition 5.1, we get

$$
\beta_{\Phi_{t}} \mathbb{E}_{\Phi_{t}}^{0} \sum_{X \in \Phi_{t}} f(\|X\|)=\frac{1}{b^{d}} \mathbb{E} \sum_{X \in \Phi_{t} \cap C_{0}} \sum_{Y \neq X \in \Phi_{t}} f(\|X-Y\|)
$$




$$
\begin{aligned}
\leq & \frac{K}{b^{d}} \mathbb{E} \Phi_{t}\left(C_{0}\right)^{2} \\
& +\frac{1}{b^{d}} \mathbb{E} \sum_{X \in \Phi_{t} \cap C_{0}} \sum_{i \neq 0} \sum_{Y \in \Phi_{t} \cap C_{i}} f(\|X-Y\|) \\
\leq & \frac{K}{b^{d}} \mathbb{E} U_{t}(0)^{2}+\frac{\left(\mathbb{E} U_{t}(0)\right)^{2}}{b^{d}} \sum_{i \neq 0} f\left(d_{i}+\right),
\end{aligned}
$$

where $K$ is the upper-bound on $f$ (Assumption 3), $U(0)$ is the elementary mutual service process defined in the proof of Proposition 5.1, $d_{i}$ is the distance from $C_{i}$ to $C_{0}$, and $d_{i}+$ stands for the right-hand limit (to handle the case where $d_{i}=0$ ). There exists a constant $H>1$ and a ball $B$ centered in the origin such that for all $i$ with $C_{i}$ not included in $B$ and for all $x \in C_{i},\|x\| \leq H d_{i}$. If $\nu_{d}$ denotes the volume of a unit ball, this in turn implies that

$$
\frac{1}{b^{d}} \sum_{i \neq 0, C_{i} \notin B} f\left(d_{i}\right) \leq \int_{\mathbb{R}^{d}} f\left(\frac{\|x\|}{H}\right) d x=d \nu_{d} \int_{r>0} f\left(\frac{r}{H}\right) r^{d-1} d r=H^{d} a<\infty .
$$

The proof of the first statement is then concluded from the second statement of Proposition 5.1 and from the fact that the moments of $U_{t}(0)$ are uniformly bounded.

Let $H_{t}=\sum_{i \neq 0} f\left(d_{i}+\right) U_{i}(t)$. For all $k>1$,

$$
\begin{aligned}
\beta_{\Phi_{t}} \mathbb{E}_{\Phi_{t}}^{0}\left(\sum_{X \in \Phi_{t}} f(\|X\|)\right)^{k} & =\frac{1}{b^{d}} \mathbb{E} \sum_{X \in \Phi_{t} \cap C_{0}}\left(\sum_{Y \neq X \in \Phi_{t}} f(\|X-Y\|)\right)^{k} \\
& \leq \frac{1}{b^{d}} \mathbb{E}\left(U_{t}(0)\left(K U_{t}(0)+H_{t}\right)^{k}\right) \\
& =\frac{1}{b^{d}} \sum_{p=0}^{k}\left(\begin{array}{c}
k \\
p
\end{array}\right) K^{p} \mathbb{E} U_{t}(0)^{p+1} \mathbb{E} H_{t}^{k-p}
\end{aligned}
$$

so that it is enough to prove that $\mathbb{E} H_{t}^{p}$ is bounded above uniformly in $t$ and $p \leq k$. But this follows immediately from the uniform bounds on $\mathbb{E} U_{t}(i)^{n}, i, t \geq 0$ and $n \leq k$ (Proposition 5.1) and from the finiteness of $a$, which implies the finiteness of the series $\sum_{i \neq 0} f^{k}\left(d_{i}\right)$.

The results of the last lemma extend to the point process $\Phi_{t}^{\prime}=\Phi_{t, \mathcal{Z}_{0}}$ built by Construct for all thinned Poisson initial conditions.

\subsection{Positivity}

We now prove that for all finite $t$, the densities of all our point processes are positive. We denote by $\mathcal{R}_{t}$ (resp. $\mathcal{Z}_{t}, \mathcal{A}_{t}$ and $\mathcal{S}_{t}$ ) the stationary point process of regular points (resp. Z points, A points and special points) built by Construct $^{Z}$ at time $t$. For each of these point processes, say $\mathcal{X}_{t}$, we denote its intensity by $\beta \mathcal{X}_{t}$. 
Lemma 5.2. Make Assumptions $0-2$, and let $\beta_{\mathcal{Z}_{0}}>0$. Then $\beta_{\mathcal{X}_{t}}>0$ for all finite $t$, with $\mathcal{X}_{t}=\mathcal{R}_{t}, \mathcal{Z}_{t}, \mathcal{A}_{t}, \mathcal{S}_{t}$.

Proof. Let $t$ be fixed and finite. We start with $\mathcal{X}_{t}=\mathcal{Z}_{t}$. Let $z$ be a typical point of $\mathcal{Z}_{0}$. The total number of points of $\Psi(0, t)$ that have a connection to $z$ that takes place before time $t$ is (stochastically) bounded from above by a Poisson random variable with parameter

$$
\int_{\mathbb{R}^{d}}\left(1-e^{-f(\|x-z\|)}\right) \lambda t d x \leq \lambda t a .
$$

The total number of points of $\mathcal{Z}_{0}$ that have a connection to $z$ is also finite by assumption (Item 2 in Subsection 3.2). Hence the probability that all duels involving $z$ and taking place before time $t$ are oriented in such a way that $z$ survives is positive. This shows that $\beta_{\mathcal{Z}_{t}}>0$ and also that $\beta_{S_{t}}>0$.

In order to prove the result for $\mathcal{X}_{t}=\mathcal{A}_{t}$, we pick $\epsilon<t$ and we use arguments similar to those above to show that the probability that (1) $z$ survives until time $t-\epsilon ;(2)$ $\Psi(t-\epsilon, t)$ brings one arrival which kills $z$ (which becomes an A point); and (3) the latter survives until time $t$, is positive.

In order to prove the result for $\mathcal{X}_{t}=\mathcal{R}_{t}$, we pick $\epsilon<t$ and we look at the arrivals of $\Psi(0, \epsilon)$ in each box $C_{i}=i+[0,1)^{d}$, where $k$ ranges over $\mathbb{Z}^{d}$. For those boxes that have at least one arrival, pick the first of them. This defines a point process. For a typical point of this point process, say $r$, we use arguments similar to those above to show that the probability that $r$ survives until time $t$ is positive. This shows that $\beta_{\mathcal{R}_{t}}>0$.

Remark 5.2. It follows from the last lemma and from 2. in Section 4.3 that the densities $\beta_{\Phi_{t}}$ and $\beta_{\Phi_{t}^{\prime}}$ are also positive for all finite $t$.

\section{Differential equations for transient moment measures}

The setting of this section is the same as that of Section 4, with the empty and augmented initial conditions. We complement the result on the finiteness of the special points stemming from a single point (Theorem 4.2) by a set of differential equations on the densities and higher order moment measures of nodes of all types. These equations will be needed in the coupling from the past arguments of the next section.

We assume that the augmentation $\mathcal{Z}_{0}$ is a motion invariant point process satisfying the assumptions of Subsection 3.2. The default setting is that Assumptions 0-3 hold.

\subsection{ODEs for densities}

\subsubsection{Construct}

Let $\beta_{\Phi^{\prime} t}$ denote the density of the point process $\Phi_{t}^{\prime}$ built by Construct for the initial condition $\mathcal{Z}_{0}$. Let $\mathbb{E}_{\Phi_{t}^{\prime}}^{0}$ denote its Palm probability (since $\beta_{\Phi^{\prime}{ }_{t}}>0$, see Remark 5.2, the latter is well defined). For all $x \in \mathbb{R}^{d}$, let

$$
\pi_{\Phi_{t}^{\prime}}(x)=\sum_{X \in \Phi_{t}^{\prime}} f(\|X-x\|) \text {. }
$$


This quantity can intuitively be interpreted as the death pressure exerted by $\Phi_{t}^{\prime}$ at $x$. The death rate of a typical node living at time $t$ is $\mathbb{E}_{\Phi_{t}^{\prime}}^{0} \pi_{\Phi_{t}^{\prime}}(0)$. The following equation is proved in Appendix 9.1.

$$
\frac{d}{d t} \beta_{\Phi^{\prime} t}=\lambda-\beta_{\Phi_{t}^{\prime}} \mathbb{E}_{\Phi_{t}^{\prime}}^{0} \pi_{\Phi_{t}^{\prime}}(0)
$$

From Proposition 5.1 and Property 1 at the end of Section 5.1, the term $\beta_{\Phi_{t}^{\prime}} \mathbb{E}_{\Phi_{t}^{\prime}}^{0} \pi_{\Phi_{t}^{\prime}}(0)$ that we find on the R.H.S. of this differential equation is uniformly bounded in $t$.

From the fact that $\beta_{\Phi_{t}^{\prime}}$ is uniformly bounded and from (6.2), we also get that

$$
\frac{1}{t} \int_{0}^{t} \beta_{\Phi_{u}^{\prime}} \mathbb{E}_{\Phi_{u}^{\prime}}^{0} \pi_{\Phi_{u}^{\prime}}(0) d u=\lambda+o(1)
$$

as $t$ tends to infinity.

\subsubsection{Construct ${ }^{Z}$}

For all $t>0$, for each of the point processes $\mathcal{X}_{t}=\mathcal{R}_{t}, \mathcal{Z}_{t}, \mathcal{A}_{t}$ or $\mathcal{S}_{t}$, since $\beta_{X_{t}}>0$ (Lemma 5.2), the Palm probability $\mathbb{E}_{\mathcal{X}_{t}}^{0}$ w.r.t. $\mathcal{X}_{t}$ is well defined.

Assuming $\mathcal{X}_{t}$ is the point process of nodes that interact with a node located at $x$, we define the death pressure exerted by the nodes of $\mathcal{X}_{t}$ on $x$ as

$$
\pi_{\mathcal{X}_{t}}(x):=\sum_{X \in \mathcal{X}_{t}} f(\|X-x\|), \quad \pi_{\mathcal{X}_{t}}:=\pi_{\mathcal{X}_{t}}(0) .
$$

Since $\mathrm{Z}$ points and A points do not interact, we refine this definition in the case of $\mathcal{S}_{t}$ as follows:

$$
\pi_{\mathcal{S}_{t}}(x)=\left\{\begin{array}{l}
\sum_{y \in \mathcal{Z}_{t}} f(|x-y|) \text { if } x \in \mathcal{Z}_{t} \\
\sum_{y \in \mathcal{A}_{t}} f(|x-y|) \text { if } x \in \mathcal{A}_{t} \\
\sum_{y \in \mathcal{S}_{t}} f(|x-y|) \text { otherwise }
\end{array}\right.
$$

This must be taken into account when working with the Palm probability of special points, since the point at origin may be of either type. Consequently, the general relation

$$
\mathbb{E}_{\mathcal{S}_{t}}^{0}=\frac{\beta_{\mathcal{Z}_{t}}}{\beta_{\mathcal{S}_{t}}} \mathbb{E}_{\mathcal{Z}_{t}}^{0}+\frac{\beta_{\mathcal{A}_{t}}}{\beta_{\mathcal{S}_{t}}} \mathbb{E}_{\mathcal{A}_{t}}^{0}
$$

gives, for example,

$$
\mathbb{E}_{\mathcal{S}_{t}}^{0} \pi_{\mathcal{S}_{t}}=\frac{\beta_{\mathcal{Z}_{t}}}{\beta_{\mathcal{S}_{t}}} \mathbb{E}_{\mathcal{Z}_{t}}^{0} \pi_{\mathcal{Z}_{t}}+\frac{\beta_{\mathcal{A}_{t}}}{\beta_{\mathcal{S}_{t}}} \mathbb{E}_{\mathcal{A}_{t}}^{0} \pi_{\mathcal{A}_{t}}
$$

Notice that the mass transport principle (see [15] or Appendix 9.2) implies that

$$
\begin{aligned}
\beta_{\mathcal{Z}_{t}} \mathbb{E}_{\mathcal{Z}_{t}}^{0} \pi_{\mathcal{R}_{t}} & =\beta_{\mathcal{R}_{t}} \mathbb{E}_{\mathcal{R}_{t}}^{0} \pi_{\mathcal{Z}_{t}} \\
\beta_{\mathcal{A}_{t}} \mathbb{E}_{\mathcal{A}_{t}}^{0} \pi_{\mathcal{R}_{t}} & =\beta_{\mathcal{R}_{t}} \mathbb{E}_{\mathcal{R}_{t}}^{0} \pi_{\mathcal{A}_{t}} .
\end{aligned}
$$


Lemma 6.1. Under the foregoing assumptions,

$$
\begin{aligned}
\frac{d}{d t} \beta_{\mathcal{Z}_{t}} & =-\beta_{\mathcal{Z}_{t}} \mathbb{E}_{\mathcal{Z}_{t}}^{0} \pi_{\mathcal{Z}_{t}+\mathcal{R}_{t}}+\beta_{\mathcal{R}_{t}} \mathbb{E}_{\mathcal{R}_{t}}^{0} \pi_{\mathcal{A}_{t}} \\
\frac{d}{d t} \beta_{\mathcal{A}_{t}} & =-\beta_{\mathcal{A}_{t}} \mathbb{E}_{\mathcal{A}_{t}}^{0} \pi_{\mathcal{A}_{t}+\mathcal{R}_{t}}+\beta_{\mathcal{R}_{t}} \mathbb{E}_{\mathcal{R}_{t}}^{0} \pi_{\mathcal{Z}_{t}} \\
\frac{d}{d t} \beta_{\mathcal{R}_{t}} & =\lambda-\beta_{\mathcal{R}_{t}} \mathbb{E}_{\mathcal{R}_{t}}^{0} \pi_{\mathcal{R}_{t}+\mathcal{Z}_{t}+\mathcal{A}_{t}},
\end{aligned}
$$

where all the terms found on the right hand sides of these differential equations are uniformly bounded in $t$.

Proof. By arguments similar to those of Lemma 5.1, both

$$
\beta_{\mathcal{R}_{t}} \mathbb{E}_{\mathcal{R}_{t}}^{0} \pi_{\mathcal{R}_{t}+\mathcal{Z}_{t}}+\beta_{\mathcal{Z}_{t}} \mathbb{E}_{\mathcal{Z}_{t}}^{0} \pi_{\mathcal{R}_{t}+\mathcal{Z}_{t}}
$$

and

$$
\beta_{\mathcal{R}_{t}} \mathbb{E}_{\mathcal{R}_{t}}^{0} \pi_{\mathcal{R}_{t}+\mathcal{A}_{t}}+\beta_{\mathcal{A}_{t}} \mathbb{E}_{\mathcal{A}_{t}}^{0} \pi_{\mathcal{R}_{t}+\mathcal{A}_{t}}
$$

are uniformly bounded in $t$, which in turn implies that all the terms found on the right hand sides of the differential equations are uniformly bounded in $t$.

The average death rate of a $\mathrm{Z}$ point is $\mathbb{E}_{\mathcal{Z}_{t}}^{0} \pi_{\mathcal{Z}_{t}+\mathcal{R}_{t}}$. The rate at which a regular point is transformed into a $\mathrm{Z}$ point is $\mathbb{E}_{\mathcal{R}_{t}}^{0} \pi_{\mathcal{A}_{t}}$. The equations are then obtained by arguments similar to those used in Appendix 9.1 to prove (6.2).

Notice that (6.11) can be rewritten as

$$
\frac{d}{d t} \beta_{\mathcal{R}_{t}}=\lambda-\beta_{\mathcal{R}_{t}} \mathbb{E}_{\mathcal{R}_{t}}^{0} \pi_{\mathcal{R}_{t}}-\beta_{\mathcal{Z}_{t}} \mathbb{E}_{\mathcal{Z}_{t}}^{0} \pi_{\mathcal{R}_{t}}-\beta_{\mathcal{A}_{t}} \mathbb{E}_{\mathcal{A}_{t}}^{0} \pi_{\mathcal{R}_{t}}
$$

These equations are consistent with those established in the last subsection. When adding (6.9) and (6.11), and when using the fact that $\beta_{\Phi_{t}^{\prime}}=\beta_{\mathcal{Z}_{t}}+\beta_{\mathcal{R}_{t}}$, we get

$$
\begin{aligned}
\frac{d}{d t} \beta_{\Phi_{t}^{\prime}} & =\lambda-\beta_{\mathcal{R}_{t}} \mathbb{E}_{\mathcal{R}_{t}}^{0} \pi_{\mathcal{R}_{t}}-\beta_{\mathcal{Z}_{t}} \mathbb{E}_{\mathcal{Z}_{t}}^{0} \pi_{\mathcal{Z}_{t}}-2 \beta_{\mathcal{R}_{t}} \mathbb{E}_{\mathcal{R}_{t}}^{0} \pi_{\mathcal{Z}_{t}} \\
& =\lambda-\beta_{\Phi_{t}^{\prime}} \mathbb{E}_{\Phi_{t}^{\prime}}^{0} \pi_{\Phi_{t}^{\prime}}
\end{aligned}
$$

which is (6.2).

When adding (6.9) and (6.10) and when using the relation (6.6), we get:

Lemma 6.2. Under the foregoing assumptions,

$$
\frac{d}{d t} \beta_{\mathcal{S}_{t}}=-\beta_{\mathcal{S}_{t}} \mathbb{E}_{\mathcal{S}_{t}}^{0} \pi_{\mathcal{S}_{t}}
$$

Hence

$$
\beta_{\mathcal{S}_{t}}=\beta_{\mathcal{S}_{0}} \exp \left(-\int_{0}^{t} \mathbb{E}_{\mathcal{S}_{u}}^{0} \pi_{\mathcal{S}_{u}} d u\right)
$$

It follows from (6.14) that $\beta_{\mathcal{S}_{t}}$ decreases and hence tends to a limit as $t$ tends to $\infty$. 


\subsection{ODEs for death pressures}

We recall that $\Phi_{t}^{\prime}$ denotes the point process built by Construct for the initial condition $\mathcal{Z}_{0}$.

Lemma 6.3. Under Assumptions 0-3, we have

$$
\frac{d}{d t}\left(\beta_{\Phi_{t}^{\prime}} \mathbb{E}_{\Phi_{t}^{\prime}}^{0} \pi_{\Phi_{t}^{\prime}}\right)=2 \lambda a \beta_{\Phi_{t}^{\prime}}-2 \beta_{\Phi_{t}^{\prime}} \mathbb{E}_{\Phi_{t}^{\prime}}^{0} \pi_{\Phi_{t}^{\prime}}^{2}
$$

Proof. For all Borel sets $C$, let

$$
\Pi_{\Phi_{t}^{\prime}}\left(\Phi_{t}^{\prime} \cap C\right)=\sum_{X \in \Phi_{t}^{\prime} \cap C} \pi_{\Phi_{t}^{\prime}}(X)
$$

with $\pi_{\Phi_{t}^{\prime}}(\cdot)$ defined in (6.4). By Lemma 5.1, $\Pi_{\Phi_{t}^{\prime}}\left(\Phi_{t}^{\prime} \cap C\right)$ is integrable, and it can be compensated to a martingale by subtracting the continuous process

$$
\begin{aligned}
& \int_{0}^{t}\left[\lambda \int_{C} \pi_{\Phi_{s}^{\prime}}(y) \mathrm{d} y+\lambda \int_{\mathbb{R}^{d}} \sum_{X \in \Phi_{s}^{\prime}} f(|X-y|) \mathrm{d} y\right. \\
& \left.-\sum_{X \in \Phi_{s}^{\prime} \cap C} \pi_{\Phi_{s}^{\prime}}(X) \cdot \pi_{\Phi_{s}^{\prime}}(X)-\sum_{Y \in \Phi_{s}^{\prime}} \pi_{\Phi_{s}^{\prime}}(Y) \sum_{X \in \Phi_{s}^{\prime} \cap C} f(|X-Y|)\right] \mathrm{d} s .
\end{aligned}
$$

Taking expectations, using Fubini, the translation invariance of $\Phi_{s}^{\prime}$, and differentiating (similarly to the simpler case of Appendix 9.1) we obtain the differential equation

$$
\begin{aligned}
\frac{d}{d t} \mathbb{E}_{\Phi_{t}^{\prime}}\left(\Phi_{t}^{\prime} \cap C\right)= & 2 \lambda a \mathbb{E}\left|\Phi_{t}^{\prime} \cap C\right| \\
& -\mathbb{E} \sum_{X \in \Phi_{t}^{\prime} \cap C} \pi_{\Phi_{t}^{\prime}}^{2}(X) \\
& -\mathbb{E} \sum_{Y \in \Phi_{t}^{\prime}} \pi_{\Phi_{t}^{\prime} \cap C}(Y) \pi_{\Phi_{t}^{\prime}}(Y) .
\end{aligned}
$$

The rationale is the following: $\Pi_{\Phi_{t}^{\prime}}\left(\Phi_{t}^{\prime} \cap C\right)$ represents the pressure exerted by $\Phi_{t}^{\prime}$ on $\Phi_{t}^{\prime} \cap C$. The reasons for this pressure to change with time are:

- A new point can be born anywhere from the Poisson rain process. For each $X \in \Phi_{t-}^{\prime} \cap C$, the average pressure increase per time unit due to arrivals is $\lambda a$. In the case where that point is born in $C$, which happens with intensity $\lambda|C|$, it meets in average a pressure of strength $\beta_{\Phi_{t}^{\prime}} a$, which is added to the total pressure. Using $\beta_{\Phi_{t}^{\prime}}|C|=\mathbb{E}\left|\Phi_{t}^{\prime} \cap C\right|$, the two effects give the first term of the R.H.S.

- Each $X$ in $\Phi_{t}^{\prime} \cap C$ can be killed by another point. This happens with intensity $\pi_{\Phi_{t}^{\prime}}(X)$. The death of $X$ will decrease the total pressure by $\pi_{\Phi_{t}^{\prime}}(X)$, hence the second term. This process also removes some pressure to the remaining points from $\Phi_{t}^{\prime} \cap C$, but this effect is considered in the third term. 
- Each $Y$ in $\Phi_{t}^{\prime}$ can be killed. This happens with intensity $\pi_{\Phi_{t}^{\prime}}(Y)$. The death of $Y$ will remove the pressure $\pi_{\Phi_{t}^{\prime} \cap C}(Y)$ between $Y$ and $\Phi_{t}^{\prime} \cap C$, hence the third term.

By standard arguments, we have

$$
\mathbb{E} \Pi_{\Phi_{t}^{\prime}}\left(\Phi_{t}^{\prime} \cap C\right)=\beta_{\Phi_{t}^{\prime}}|C| \mathbb{E}_{\Phi_{t}^{\prime}}^{0} \pi_{\Phi_{t}^{\prime}}
$$

and

$$
\mathbb{E} \sum_{X \in \Phi_{t}^{\prime} \cap C} \pi_{\Phi_{t}^{\prime}}^{2}(X)=\beta_{\Phi_{t}^{\prime}}|C| \mathbb{E}_{\Phi_{t}^{\prime}}^{0} \pi_{\Phi_{t}^{\prime}}^{2}
$$

Using the mass transport principle (cf Appendix 9.2), we have

$$
\mathbb{E} \sum_{Y \in \Phi_{t}^{\prime}} \pi_{\Phi_{t}^{\prime} \cap C}(Y) \pi_{\Phi_{t}^{\prime}}(Y)=\beta_{\Phi_{t}^{\prime}}|C| \mathbb{E}_{\Phi_{t}^{\prime}}^{0} \pi_{\Phi_{t}^{\prime}}^{2} .
$$

Hence (6.18) can be rewritten as indicated in the lemma.

Proposition 6.1. Under the assumptions of this Section, the following differential equations hold for the pressure of regulars on specials:

$$
\begin{aligned}
& \frac{d}{d t}\left(\beta_{\mathcal{Z}_{t}} \mathbb{E}_{\mathcal{Z}_{t}}^{0}\left[\pi_{\mathcal{R}_{t}}\right]\right)=\beta_{\mathcal{R}_{t}} \mathbb{E}_{\mathcal{R}_{t}}^{0}\left[\pi_{\mathcal{R}_{t}} \pi_{\mathcal{A}_{t}}\right]+\lambda \beta_{\mathcal{Z}_{t}} a \\
& \quad-\beta_{\mathcal{Z}_{t}} \mathbb{E}_{\mathcal{Z}_{t}}^{0}\left[\pi_{\mathcal{R}_{t}} \pi_{\mathcal{R}_{t}+\mathcal{Z}_{t}}\right]-\beta_{\mathcal{R}_{t}} \mathbb{E}_{\mathcal{R}_{t}}^{0}\left[\pi_{\mathcal{Z}_{t}} \pi_{\mathcal{R}_{t}+\mathcal{Z}_{t}+\mathcal{A}_{t}}\right]
\end{aligned}
$$

as well as the symmetrical one (i.e. that for $\beta_{\mathcal{A}_{t}} \mathbb{E}_{\mathcal{A}_{t}}^{0}\left[\pi_{\mathcal{R}_{t}}\right]$ ). In addition

$$
\frac{d}{d t}\left(\beta_{\mathcal{S}_{t}} \mathbb{E}_{\mathcal{S}_{t}}^{0}\left[\pi_{\mathcal{R}_{t}}\right]\right)=\lambda \beta_{\mathcal{S}_{t}} a-\beta_{\mathcal{S}_{t}} \mathbb{E}_{\mathcal{S}_{t}}^{0}\left[\pi_{\mathcal{R}_{t}} \pi_{\mathcal{R}_{t}+\mathcal{S}_{t}}\right]-\beta_{\mathcal{R}_{t}} \mathbb{E}_{\mathcal{R}_{t}}^{0}\left[\left(\pi_{\mathcal{S}_{t}}\right)^{2}\right]
$$

For the pressure of specials on specials, we have

$$
\frac{d}{d t}\left(\beta_{\mathcal{Z}_{t}} \mathbb{E}_{\mathcal{Z}_{t}}^{0}\left[\pi_{\mathcal{Z}_{t}}\right]\right)=2 \beta_{\mathcal{R}_{t}} \mathbb{E}_{\mathcal{R}_{t}}^{0}\left[\pi_{\mathcal{Z}_{t}} \pi_{\mathcal{A}_{t}}\right]-2 \beta_{\mathcal{Z}_{t}} \mathbb{E}_{\mathcal{Z}_{t}}^{0}\left[\pi_{\mathcal{Z}_{t}} \pi_{\mathcal{Z}_{t}+\mathcal{R}_{t}}\right]
$$

as well as the symmetrical one (i.e. that for $\beta_{\mathcal{A}_{t}} \mathbb{E}_{\mathcal{A}_{t}}^{0}\left[\pi_{\mathcal{A}_{t}}\right]$ ). In addition

$$
\frac{d}{d t}\left(\beta_{\mathcal{S}_{t}} \mathbb{E}_{\mathcal{S}_{t}}^{0}\left[\pi_{\mathcal{S}_{t}}\right]\right)=4 \beta_{\mathcal{R}_{t}} \mathbb{E}_{\mathcal{R}_{t}}^{0}\left[\pi_{\mathcal{Z}_{t}} \pi_{\mathcal{A}_{t}}\right]-2 \beta_{\mathcal{S}_{t}} \mathbb{E}_{\mathcal{S}_{t}}^{0}\left[\pi_{\mathcal{S}_{t}} \pi_{\mathcal{S}_{t}+\mathcal{R}_{t}}\right]
$$

Finally, for the pressure of regulars on regulars, we have

$$
\frac{d}{d t}\left(\beta_{\mathcal{R}_{t}} \mathbb{E}_{\mathcal{R}_{t}}^{0}\left[\pi_{\mathcal{R}_{t}}\right]\right)=\lambda \beta_{\mathcal{R}_{t}} a-2 \beta_{\mathcal{R}_{t}} \mathbb{E}_{\mathcal{R}_{t}}^{0}\left[\pi_{\mathcal{R}_{t}} \pi_{\mathcal{R}_{t}+\mathcal{S}_{t}}\right]
$$

All terms in the RHSs of these differential equations are uniformly bounded. 
Proof. The last property is obtained by the same arguments as for densities (see Section 5.1).

For all Borel sets $C_{1}, C_{2}$ and for all point processes $\mathcal{X}_{t}, \mathcal{Y}_{t}$, consider

$$
\Pi_{\mathcal{Y}_{t} \cap C_{2}}\left(\mathcal{X}_{t} \cap C_{1}\right)=\sum_{X \in \mathcal{X}_{t} \cap C_{1}} \pi_{\mathcal{Y}_{t} \cap C_{2}}(X) .
$$

We remind the pressure analogy used in the proof of (6.16): $\Pi_{\mathcal{Y}_{t} \cap C_{2}}\left(\mathcal{X}_{t} \cap C_{1}\right)$ can be seen as the death pressure exerted by $\mathcal{Y}_{t} \cap C_{2}$ on $\mathcal{X}_{t} \cap C_{1}$. Note that because of the symmetry of the processes, it is also the pressure exerted by $\mathcal{X}_{t} \cap C_{1}$ on $\mathcal{Y}_{t} \cap C_{2}$.

We first prove (6.20). For all sets $C$, we have

$$
\begin{aligned}
\frac{d}{d t} \mathbb{E}_{\mathcal{R}_{t}}\left(\mathcal{Z}_{t} \cap C\right)= & \mathbb{E} \sum_{X \in \mathcal{R}_{t} \cap C} \pi_{\mathcal{R}_{t}}(X) \pi_{\mathcal{A}_{t}}(X) \\
& +\lambda a \mathbb{E}\left|\mathcal{Z}_{t} \cap C\right| \\
& -\mathbb{E} \sum_{X \in \mathcal{Z}_{t} \cap C} \pi_{\mathcal{R}_{t}}(X) \pi_{\mathcal{R}_{t}+\mathcal{Z}_{t}}(X) \\
& -\mathbb{E} \sum_{X \in \mathcal{R}_{t}} \pi_{\mathcal{Z}_{t} \cap C}(X) \pi_{\mathcal{R}_{t}+\mathcal{Z}_{t}+\mathcal{A}_{t}}(X) .
\end{aligned}
$$

The reason is the following: $\Pi_{\mathcal{R}_{t}}\left(\mathcal{Z}_{t} \cap C\right)$ represents the pressure exerted by $\mathcal{R}_{t}$ on $\mathcal{Z}_{t} \cap C$. The reasons for this pressure to change with time are:

- A regular point $X \in \mathcal{R}_{t} \cap C$ can be turned into a new $\mathrm{Z}$ point due to a regularA point interaction. For each $X \in \mathcal{R}_{t} \cap C$, this happens with intensity $\pi_{\mathcal{A}_{t}}(X)$. The newborn $\mathrm{Z}$ point will experience a pressure $\pi_{\mathcal{R}_{t}}(X)$ (we recall the convention $f(0)=0)$; hence the first term in (6.26). This process also removes $X$ as a regular point, but this effect is considered in the fourth term.

- A new regular point can be born from the Poisson rain process. For each $Z \in$ $\mathcal{Z}_{t} \cap C$, the average pressure increase per time unit due to arrivals is $\lambda a$, hence the second term.

- Each Z point $X \in \mathcal{Z}_{t} \cap C$ can be killed by a regular point or a $\mathrm{Z}$ point. This happens with intensity $\pi_{\mathcal{R}_{t}+\mathcal{Z}_{t}}(X)$. The death of $X$ will decrease the total pressure by $\pi_{\mathcal{R}_{t}}(X)$, hence the third term.

- Each regular point $X \in \mathcal{R}_{t}$ can be killed by anyone (regular or special). This happens with intensity $\pi_{\mathcal{R}_{t}+\mathcal{Z}_{t}+\mathcal{A}_{t}}(X)$. The death of $X$ will remove the pressure $\pi_{\mathcal{Z}_{t} \cap C}(X)$ between $X$ and $\mathcal{Z}_{t} \cap C$, hence the last term.

Each term in (6.26) including the one differentiated are uniformly bounded for the same reasons as those used in the proof of Lemma 5.1 (tightness of the quantities of interest for both initial conditions). From the very definition of Palm probability, we can rewrite the term which is differentiated in (6.26) as

$$
\mathbb{E} \Pi_{\mathcal{R}_{t}}\left(\mathcal{Z}_{t} \cap C\right)=\beta_{\mathcal{Z}_{t}}|C| \mathbb{E}_{\mathcal{Z}_{t}}^{0}\left[\pi_{\mathcal{R}_{t}}\right]
$$


and the first and third terms on the R.H.S. as

$$
\begin{aligned}
\mathbb{E} \sum_{X \in \mathcal{R}_{t} \cap C} \pi_{\mathcal{R}_{t}}(X) \pi_{\mathcal{A}_{t}}(X) & =\beta_{\mathcal{R}_{t}}|C| \mathbb{E}_{\mathcal{R}_{t}}^{0}\left[\pi_{\mathcal{R}_{t}} \pi_{\mathcal{A}_{t}}\right] \\
\mathbb{E} \sum_{X \in \mathcal{Z}_{t} \cap C} \pi_{\mathcal{R}_{t}}(X) \pi_{\mathcal{R}_{t}+\mathcal{Z}_{t}}(X) & =\beta_{\mathcal{Z}_{t}}|C| \mathbb{E}_{\mathcal{Z}_{t}}^{0}\left[\pi_{\mathcal{R}_{t}} \pi_{\mathcal{R}_{t}+\mathcal{Z}_{t}}\right],
\end{aligned}
$$

respectively. In addition, we show in Appendix 9.2 that the following identity holds for the fourth term:

$$
\mathbb{E} \sum_{X \in \mathcal{R}_{t}} \pi_{\mathcal{Z}_{t} \cap C}(X) \pi_{\mathcal{R}_{t}+\mathcal{Z}_{t}+\mathcal{A}_{t}}(X)=\mathbb{E} \sum_{X \in \mathcal{R}_{t} \cap C} \pi_{\mathcal{Z}_{t}}(X) \pi_{\mathcal{R}_{t}+\mathcal{Z}_{t}+\mathcal{A}_{t}}(X)
$$

Hence

$$
\mathbb{E} \sum_{X \in \mathcal{R}_{t}} \pi_{\mathcal{Z}_{t} \cap C}(X) \pi_{\mathcal{R}_{t}+\mathcal{Z}_{t}+\mathcal{A}_{t}}(X)=\beta_{\mathcal{R}_{t}}|C| \mathbb{E}_{\mathcal{R}_{t}}^{0}\left[\pi_{\mathcal{Z}_{t}} \pi_{\mathcal{R}_{t}+\mathcal{Z}_{t}+\mathcal{A}_{t}}\right]
$$

We get (6.20) when dividing (6.26) by $|C|$. The other equations are obtained in the same way.

Here are a few observations on these equations. Consider e.g. (6.21). The only positive term in the RHS of this equation is $\lambda \beta_{\mathcal{S}_{t}} a$. In particular, the positive term $\beta_{\mathcal{R}_{t}} \mathbb{E}_{\mathcal{R}_{t}}^{0}\left[\pi_{\mathcal{R}_{t}} \pi_{\mathcal{S}_{t}}\right]$ (contamination of an $R$ by an $S$ ) is nullified. The reason is obvious if one considers the death of a typical $R \in \mathcal{R}_{t}$. $R$ undergoes a pressure $\pi_{\mathcal{R}_{t}}(R)$ from $\mathcal{R}_{t}$ and $\pi_{\mathcal{S}_{t}}(R)$ from $\mathcal{S}_{t}$. If the killing comes from the pressure of $\mathcal{S}_{t}$, a new special is created in $R$ and the pressure from $\mathcal{R}_{t}$ will be added to the pressure between $\mathcal{S}_{t}$ and $\mathcal{R}_{t}{ }^{12}$. Conversely, if the killing comes from the pressure of $\mathcal{R}_{t}, R$ is removed and its pressure from $\mathcal{S}_{t}$ is subtracted.

It is not difficult to check the following consistency property: when adding twice (6.20), (6.22) and (6.24), we get back (6.16) as expected.

Proposition 6.2. Under the foregoing assumptions, both $\mathbb{E}_{\mathcal{S}_{t}}^{0} \pi_{\mathcal{R}_{t}}$ and $\mathbb{E}_{\mathcal{S}_{t}}^{0} \pi_{\mathcal{S}_{t}}$ are uniformly bounded with respect to $t$.

Proof. By adding twice (6.21) and (6.23), we get

$$
\begin{aligned}
\frac{d}{d t}\left(\beta_{\mathcal{S}_{t}} \mathbb{E}_{\mathcal{S}_{t}}^{0}\left[2 \pi_{\mathcal{R}_{t}}+\pi_{\mathcal{S}_{t}}\right]\right)= & 2 \lambda \beta_{\mathcal{S}_{t}} a-2 \beta_{\mathcal{S}_{t}} \mathbb{E}_{\mathcal{S}_{t}}^{0}\left[\left(\pi_{\mathcal{R}_{t}}+\pi_{\mathcal{S}_{t}}\right)^{2}\right] \\
& -2 \beta_{\mathcal{R}_{t}} \mathbb{E}_{\mathcal{R}_{t}}^{0}\left[\left(\pi_{\mathcal{A}_{t}}+\pi_{\mathcal{Z}_{t}}\right)^{2}\right]+4 \beta_{\mathcal{R}_{t}} \mathbb{E}_{\mathcal{R}_{t}}^{0}\left[\pi_{\mathcal{Z}_{t}} \pi_{\mathcal{A}_{t}}\right] \\
\leq & 2 \lambda \beta_{\mathcal{S}_{t}} a-2 \beta_{\mathcal{S}_{t}} \mathbb{E}_{\mathcal{S}_{t}}^{0}\left[\left(\pi_{\mathcal{R}_{t}}+\pi_{\mathcal{S}_{t}}\right)^{2}\right] .
\end{aligned}
$$

By making use of (6.14) in the last equation, we get

$$
\begin{aligned}
\frac{d}{d t} \mathbb{E}_{\mathcal{S}_{t}}^{0}\left[2 \pi_{\mathcal{R}_{t}}+\pi_{\mathcal{S}_{t}}\right] & \leq 2 \lambda a+\mathbb{E}_{\mathcal{S}_{t}}^{0}\left[\pi_{\mathcal{S}_{t}}\right] \mathbb{E}_{\mathcal{S}_{t}}^{0}\left[2 \pi_{\mathcal{R}_{t}}+\pi_{\mathcal{S}_{t}}\right]-2 \mathbb{E}_{\mathcal{S}_{t}}^{0}\left[\left(\pi_{\mathcal{R}_{t}}+\pi_{\mathcal{S}_{t}}\right)^{2}\right] \\
& \leq 2 \lambda a+\left(\mathbb{E}_{\mathcal{S}_{t}}^{0}\left[\pi_{\mathcal{S}_{t}}+\pi_{\mathcal{R}_{t}}\right]\right)^{2}-2 \mathbb{E}_{\mathcal{S}_{t}}^{0}\left[\left(\pi_{\mathcal{R}_{t}}+\pi_{\mathcal{S}_{t}}\right)^{2}\right]
\end{aligned}
$$

\footnotetext{
${ }^{12}$ Meanwhile, the pressure $\pi_{\mathcal{S}_{t}}(R)$ is also removed, hence the $-\left(\pi_{\mathcal{S}_{t}}(R)\right)^{2}$ term.
} 


$$
\leq 2 \lambda a-\left(\mathbb{E}_{\mathcal{S}_{t}}^{0}\left[\pi_{\mathcal{R}_{t}}+\pi_{\mathcal{S}_{t}}\right]\right)^{2} .
$$

Thus $\mathbb{E}_{\mathcal{S}_{t}}^{0}\left[2 \pi_{\mathcal{R}_{t}}+\pi_{\mathcal{S}_{t}}\right]$ is decreasing whenever $\mathbb{E}_{\mathcal{S}_{t}}^{0}\left[\pi_{\mathcal{R}_{t}}+\pi_{\mathcal{S}_{t}}\right]>\sqrt{2 \lambda a}$. As $\mathbb{E}_{\mathcal{S}_{t}}^{0}\left[2 \pi_{\mathcal{R}_{t}}+\right.$ $\left.\pi_{\mathcal{S}_{t}}\right]>2 \sqrt{2 \lambda a}$ implies $\mathbb{E}_{\mathcal{S}_{t}}^{0}\left[\pi_{\mathcal{R}_{t}}+\pi_{\mathcal{S}_{t}}\right]>\sqrt{2 \lambda a}$, we get

$$
\limsup _{t \rightarrow \infty} \mathbb{E}_{\mathcal{S}_{t}}^{0}\left[2 \pi_{\mathcal{R}_{t}}+\pi_{\mathcal{S}_{t}}\right] \leq 2 \sqrt{2 \lambda a} .
$$

\section{Construction of the stationary regime}

The most important result of Section 4 for the construction of the stationary regime is Theorem 4.2, which shows that the perturbation induced by any point (in a finite intensity augmentation point process) a.s. vanishes with time. If we were on a finite domain, there would be a finite number of additional points, each with an influence that vanishes in finite time and the processes with and without augmentation would hence coincide after a finite time. This would provide a natural way of proving the existence and the uniqueness of the stationary regimes through a coupling from the past construction. This is the line of thought that we follow. The main technical difficulty consists in proving that the influence of the additional points vanishes fast enough.

In this section, we assume that the initial condition is a motion-invariantly thinned homogeneous Poisson point process of finite intensity.

\subsection{Exponential decay of the density of special points}

In this subsection, we suppose that Assumptions 0-3 hold. We will use the following notation for $\sigma$-algebras:

$$
\begin{aligned}
\mathcal{D}_{t} & =\sigma\left(\mathcal{Z}_{0} \cup \Psi_{(0, t]}\right) \\
\mathcal{T}_{t} & =\sigma\left(T_{p q}: p, q \in \mathcal{Z}_{0} \cup \Psi_{(0, t]}\right) \\
\mathcal{I}_{t} & =\sigma\left(I_{p q}: p, q \in \mathcal{Z}_{0} \cup \Psi_{(0, t]}\right) \\
\mathcal{G}_{t} & =\mathcal{D}_{t} \vee \mathcal{T}_{t} \vee \mathcal{I}_{t} .
\end{aligned}
$$

Note that $\widetilde{\Phi}_{t}$, introduced in Section 4.3 , is $\mathcal{G}_{t}$-measurable.

For any special node $z \in \widetilde{\Phi}_{t}$, denote by $M_{z, t, s}$ the number of points that are offsprings of $z$ and that are still alive at time $s \geq t$ (here when $z$ kills an ordinary point, the latter becomes a first generation offspring of $z$; when this node kills another ordinary point, the latter is seen as a second generation offspring of $z$, etc). The following lemma is a direct corollary of what was already established in the proofs of Theorems 4.1 and 4.2:

Lemma 7.1. Under the assumptions of Theorem 4.1, for all special nodes $z \in \widetilde{\Phi}_{t}$ and all $s \geq t$,

$$
\mathbb{E}\left[M_{z, t, s} \mid \mathcal{G}_{t} \vee \mathcal{D}_{s} \vee \mathcal{T}_{s}\right] \leq 1
$$


For any $t>0$, any special point $z \in \widetilde{\Phi}_{t}$ and any fixed positive numbers $r$ and $\epsilon$, consider the event

$$
A_{1}=\left\{\Psi_{(t, t+\epsilon)}(B(z, r))=2\right\} .
$$

On $A_{1}$, denote by $\left(a, t_{a}\right),\left(b, t_{b}\right)$ the random locations of the two points of $\Psi_{(t, t+\epsilon)} \cap$ $B(z, r)$ and consider the events

$$
\begin{aligned}
& A_{2}=\left\{t_{a} \vee t_{b}<T_{z a}<T_{z b}<T_{a b}<t+\epsilon\right\}, \\
& A_{3}=\left\{\forall p \in\{z, a, b\}, \forall q \in \widetilde{\Phi}_{t} \cup\left[e_{1}\left(\Psi_{(t, t+\epsilon)}\right) \cap B(z, r)^{c}\right]: T_{p q}>t+\epsilon\right\},
\end{aligned}
$$

where $e_{1}(\Psi)$ denotes the projection $\mathbb{R}^{d} \times \mathbb{R} \rightarrow \mathbb{R}^{d}$. Let $G=A_{1} \cap A_{2} \cap A_{3}$. Notice that $G$ is in $\mathcal{G}_{t} \vee \mathcal{D}_{t+\epsilon} \vee \mathcal{T}_{t+\epsilon}$.

Lemma 7.2. Under Assumptions 0-3, for all $t, \epsilon>0$, and for all special points $z \in \widetilde{\Phi}_{t}$,

$$
\mathbb{E}\left[M_{z, t, t+\epsilon} \mid \mathcal{G}_{t}\right] \leq 1-\frac{1}{4} \mathbb{P}\left[G \mid \mathcal{G}_{t}\right] .
$$

Proof. On $G$, the value of $M_{z, t, t+\epsilon}$ depends only on the random variables $I_{z a}, I_{z b}$ and $I_{a b}$. If $I_{z a}=-1, z$ is killed without producing any offspring after $t$. If $I_{z a}=1$ and $I_{z b}=-1$, first $a$ becomes special and then $b$ kills $z$. If then $I_{a b}=1$, both $a$ and $b$ become special, whereas in the opposite case $z$ 's family dies out. Finally, if $I_{z a}=I_{z b}=1$, both $a$ and $b$ become special of same kind and, at time $T_{a b}$, one of them kills the other. Thus the expected value of $M_{z, t, t+\epsilon}$ on $G$ is $(4 \cdot 0+1 \cdot 2+1 \cdot 0+2 \cdot 2) / 8=\frac{3}{4}$. Hence

$$
\mathbb{E}\left[1_{G} M_{z, t, t+\epsilon} \mid \mathcal{G}_{t}\right]=\frac{3}{4} \mathbb{P}\left[G \mid \mathcal{G}_{t}\right]
$$

Similarly,

$$
\begin{aligned}
\mathbb{E}\left[1_{G^{c}} M_{z, t, t+\epsilon} \mid \mathcal{G}_{t}\right] & =\mathbb{E}\left[1_{G^{c}} \mathbb{E}\left[M_{z, t, t+\epsilon} \mid \mathcal{D}_{t+\epsilon} \vee \mathcal{T}_{t+\epsilon} \vee \mathcal{G}_{t}\right] \mid \mathcal{G}_{t}\right] \\
& \leq \mathbb{E}\left[1_{G^{c}} \mid \mathcal{G}_{t}\right]
\end{aligned}
$$

where we used Lemma 7.1. Hence

$$
\begin{aligned}
\mathbb{E}\left[M_{z, t, t+\epsilon} \mid \mathcal{G}_{t}\right] & =\mathbb{E}\left[1_{G} M_{z, t, t+\epsilon} \mid \mathcal{G}_{t}\right]+\mathbb{E}\left[1_{G^{c}} M_{z, t, t+\epsilon} \mid \mathcal{G}_{t}\right] \\
& =\frac{3}{4} \mathbb{P}\left[G \mid \mathcal{G}_{t}\right]+\mathbb{E}\left[1_{G^{c}} M_{z, t, t+\epsilon} \mid \mathcal{G}_{t}\right] \\
& \leq \frac{3}{4} \mathbb{P}\left[G \mid \mathcal{G}_{t}\right]+1-\mathbb{P}\left[G \mid \mathcal{G}_{t}\right]
\end{aligned}
$$

From classical properties of Poisson point processes, reminding that $\nu_{d}$ denotes the volume of a unit ball in $\mathbb{R}^{d}$, we get

$$
\mathbb{P}\left[G \mid \mathcal{G}_{t}\right]=\mathbb{P}\left[A_{1}\right] \frac{1}{\left(\nu_{d} r^{d} \epsilon\right)^{2}} \int_{B(z, r)} \int_{B(z, r)} \int_{[t, t+\epsilon]} \int_{[t, t+\epsilon]}
$$




$$
\begin{aligned}
& \mathbb{P}\left[u \vee v<T_{z x}<T_{z y}<T_{x y}<t+\epsilon\right] \times \\
& \mathbb{P}\left[\forall p \in\{z, x, y\}, \forall q \in \widetilde{\Phi}_{t} \cup\left[e_{1}\left(\Psi_{(t, t+\epsilon)}\right) \cap B(z, r)^{c}\right]: T_{p q}>t+\epsilon \mid \mathcal{G}_{t}\right] \\
& d x d y d u d v .
\end{aligned}
$$

Notice that $\mathbb{P}\left[A_{1}\right]$ is a constant that does not depend on $t$. Similarly, if $r$ is such that $f(2 r)>0$, then $\mathbb{P}\left[u \vee v<T_{z x}<T_{z y}<T_{x y}<t+\epsilon\right]$ is bounded from below by a constant that does not depend on $t, u, v, x, y$. From this and the independence properties of the Poisson rain after $t$ and $\mathcal{G}_{t}$, we get that for all $r$ as above, there exists a constant $0<F(r, \epsilon)<1$ such that

$$
\begin{aligned}
\mathbb{P}\left[G \mid \mathcal{F}_{t}\right] \geq F(r, \epsilon) \frac{1}{\left(\nu_{d} r^{d}\right)^{2}} \int_{B(z, r)} \int_{B(z, r)} \\
\mathbb{P}\left[\forall p \in\{z, x, y\}, \forall q \in \widetilde{\Phi}_{t}: T_{p q}^{\prime}>t+\epsilon \mid \mathcal{G}_{t}\right] d x d y,
\end{aligned}
$$

where the random variables $T_{p q}^{\prime}$ are mutually independent and $T_{p q}^{\prime}$ is $t$ plus an exponential of parameter $2 f(\|p-q\|)$.

Theorem 7.1. Under Assumptions 0-3, there exists an $\alpha>0$ such that

$$
\beta_{\mathcal{S}_{t}} \leq e^{-\alpha t}
$$

for t large enough.

Proof. From Proposition 6.2, there exists a $J<\infty$ such that

$$
\mathbb{E}_{\mathcal{S}_{t}}^{0}\left[\pi_{\mathcal{S}_{t}}(0)+\pi_{\mathcal{R}_{t}}(0)\right]<J
$$

uniformly in $t$. Since the point process $\mathcal{S}_{t}$ is spatially stationary, we can use the Campbell-Mecke formula to prove that for all $\epsilon>0$,

$$
\beta_{\mathcal{S}_{t+\epsilon}}=\beta_{\mathcal{S}_{t}} \mathbb{E}_{\mathcal{S}_{t}}^{0}\left[M_{0, t, t+\epsilon}\right]
$$

with $M_{0, t, s}$ the number of special points offspring of the origin living at time $s$, under $\mathbb{P}_{\mathcal{S}_{t}}^{0}$. From Lemma 7.2 and $(7.1)$, we get

$$
\beta_{\mathcal{S}_{t+\epsilon}} \leq \beta_{\mathcal{S}_{t}}-\beta_{\mathcal{S}_{t}} \frac{1}{4} F(r, \epsilon) \xi_{t}
$$

with

$$
\xi_{t}=\frac{1}{\left(\nu_{d} r^{d}\right)^{2}} \int_{B(0, r)} \int_{B(0, r)} \mathbb{P}_{\mathcal{S}_{t}}^{0}\left[\forall p \in\{0, x, y\}, \forall q \in \widetilde{\Phi}_{t}^{0}: T_{p q}^{\prime}>t+\epsilon\right] d x d y .
$$

Let us show that when (7.3) holds, there exists an $r$, a $\epsilon$ and constant $0<C(r, \epsilon) \leq 1$ that does not depend on $t$ and such that

$$
\xi_{t}>C(r, \epsilon)
$$


We first explain the idea of the proof of (7.4) by ignoring the conditions on $x$ and $y$.

$$
\begin{aligned}
\mathbb{P}_{\mathcal{S}_{t}}^{0}\left[T_{0 q}^{\prime}>t+\epsilon, \forall q \neq 0 \in \widetilde{\Phi}_{t}^{0}\right] & =\mathbb{E}_{\mathcal{S}_{t}}^{0}\left[\mathbb{P}_{\mathcal{S}_{t}}^{0}\left[T_{0 q}>\epsilon, \forall q \neq 0 \in \widetilde{\Phi}_{t}^{0} \mid \widetilde{\Phi}_{t}\right]\right] \\
& =\mathbb{E}_{\mathcal{S}_{t}}^{0}\left[\prod_{q \neq 0 \in \widetilde{\Phi}_{t}^{0}} e^{-2 \epsilon f(\|q\|)}\right] \\
& \geq 1-\epsilon \mathbb{E}_{\mathcal{S}_{t}}^{0}\left[\sum_{q \neq 0 \in \widetilde{\Phi}_{t}^{0}} 2 f(\|q\|)\right] \\
& =1-2 \epsilon\left(\mathbb{E}_{\mathcal{S}_{t}}^{0} \pi_{\mathcal{S}_{t}}(0)+\mathbb{E}_{\mathcal{S}_{t}}^{0} \pi_{\mathcal{R}_{t}}(0)\right) \\
& \geq 1-2 \epsilon J .
\end{aligned}
$$

We now consider $x$ and $y$ in addition to 0 . By the same arguments, we have

$$
\begin{aligned}
\mathbb{P}_{\mathcal{S}_{t}}^{0}[\forall p \in\{0, x, y\}, \forall q \in & \left.\widetilde{\Phi}_{t}^{0}: T_{p q}^{\prime}>t+\epsilon\right] \\
= & \mathbb{E}_{\mathcal{S}_{t}}^{0}\left[\prod_{q \neq 0 \in \widetilde{\Phi}_{t}^{0}} e^{-2 \epsilon(f(\|q\|)+f(\|q-x\|)+f(\|q-y\|)}\right] \\
\geq & 1-2 \epsilon\left(\mathbb{E}_{\mathcal{S}_{t}}^{0} \pi_{\mathcal{S}_{t}}(0)+\mathbb{E}_{\mathcal{S}_{t}}^{0} \pi_{\mathcal{S}_{t}}(x)+\mathbb{E}_{\mathcal{S}_{t}}^{0} \pi_{\mathcal{S}_{t}}(y)\right. \\
& \left.+\mathbb{E}_{\mathcal{S}_{t}}^{0} \pi_{\mathcal{R}_{t}}(0)+\mathbb{E}_{\mathcal{S}_{t}}^{0} \pi_{\mathcal{R}_{t}}(x)+\mathbb{E}_{\mathcal{S}_{t}}^{0} \pi_{\mathcal{R}_{t}}(y)\right) .
\end{aligned}
$$

Let us now show that, under the foregoing assumptions, if $\mathbb{E}_{\mathcal{S}_{t}}^{0} \pi_{\mathcal{S}_{t}}(0)$ is uniformly bounded, then so are $\mathbb{E}_{\mathcal{S}_{t}}^{0} \pi_{\mathcal{S}_{t}}(x)$ and $\mathbb{E}_{\mathcal{S}_{t}}^{0} \pi_{\mathcal{S}_{t}}(y)$. The initial condition satisfies the assumptions of Section 3.2 and arrivals in $(0, t)$ form a marked Poisson point process on $\mathbb{R}^{d}$. Both are motion-invariant. Motion invariance is preserved by the dynamics. Hence we have

$$
\mathbb{E}_{\mathcal{S}_{t}}^{0} \pi_{\mathcal{S}_{t}}(0)=\nu_{d} d \frac{1}{\beta_{\mathcal{S}_{t}}} \int_{r>0} f(r) \rho_{\mathcal{S}_{t}}^{[2]}(r) r^{d-1} d r
$$

where $\rho_{\mathcal{S}_{t}}^{[2]}(r)$ is the radial component of the (motion invariant) density of the reduced second moment measure of $\mathcal{S}_{t}$ (see Section 8.1 for definitions). The fact that the last function is uniformly bounded implies that $\mathbb{E}_{\mathcal{S}_{t}}^{0} \mathcal{S}_{t}(B(0, b))$ is uniformly bounded for all $b$ such that $f(b)>0$. It also implies that for all $H>1$,

$$
\nu_{d} d \frac{1}{\beta_{\mathcal{S}_{t}}} \int_{r>0} f\left(\frac{r}{H}\right) \rho_{\mathcal{S}_{t}}^{[2]}\left(\frac{r}{H}\right) r^{d-1} d r
$$

is uniformly bounded. For all $x$ with $\|x\|<b$, with $b$ such that $f(b)>0$, from monotonicity and boundedness, we have

$$
\mathbb{E}_{\mathcal{S}_{t}}^{0} \pi_{\mathcal{S}_{t}}(x) \leq K \mathbb{E}_{\mathcal{S}_{t}}^{0}\left[\mathcal{S}_{t}(B(0, b))\right]+\nu_{d} d \frac{1}{\beta_{\mathcal{S}_{t}}} \int_{r \geq \rho} f\left(\frac{r}{H}\right) \rho_{\mathcal{S}_{t}}^{[2]}\left(\frac{r}{H}\right) r^{d-1} d r
$$


with $H=\frac{b}{b-\|x\|}$, which shows that $\mathbb{E}_{\mathcal{S}_{t}}^{0} \pi_{\mathcal{S}_{t}}(x)$ is uniformly bounded. By similar arguments, since $\mathbb{E}_{\mathcal{S}_{t}}^{0} \pi_{\mathcal{R}_{t}}(0)$ is uniformly bounded, then so are $\mathbb{E}_{\mathcal{S}_{t}}^{0} \pi_{\mathcal{R}_{t}}(x)$ and $\mathbb{E}_{\mathcal{S}_{t}}^{0} \pi_{\mathcal{R}_{t}}(y)$.

Thus, thanks to the uniform boundedness of all 6 terms showing up in (7.5), one can choose an $\epsilon$ and an $r$ small enough in (7.5) for (7.4) to hold.

Hence, for all $t$,

$$
\beta_{\mathcal{S}_{t+\epsilon}} \leq \beta_{\mathcal{S}_{t}}-\beta_{\mathcal{S}_{t}} \gamma
$$

with $0<\gamma=\gamma(r, \epsilon)=\frac{1}{4} F(r, \epsilon) C(r, \epsilon)<1$.

Since the function $t \rightarrow \beta_{\mathcal{S}_{t}}$ is monotone non-increasing, for $\epsilon>0$ as defined above,

$$
\beta_{\mathcal{S}_{t}} \leq \beta_{\mathcal{S}_{\epsilon\left\lfloor\frac{t}{\epsilon}\right\rfloor}} \leq \beta_{\mathcal{S}_{0}}(1-\gamma)^{\left\lfloor\frac{t}{\epsilon}\right\rfloor}
$$

with the last inequality following from the above bound. Since $\left\lfloor\frac{t}{\epsilon}\right\rfloor \geq-1+\frac{t}{\epsilon}$, it follows that

$$
\beta_{\mathcal{S}_{t}} \leq \frac{\beta_{\mathcal{S}_{0}}}{1-\gamma}(1-\gamma)^{\frac{t}{\epsilon}}
$$

for all $t$, which concludes the proof.

Theorem 7.2. Consider two executions of Construct $^{Z}$ : that with an empty initial condition and that with a stationary and ergodic initial point process $\mathcal{Z}_{0}$ which satisfies the conditions of Subsection 3.2. Under Assumptions 0-3, for all compact sets $C$ of $\mathbb{R}^{d}$, there exists a random time $\tau(C)$ with finite expectation such that for all $t \geq \tau(C)$, these two executions coincide in $C$.

Proof. Denote by $N_{t}=\mathcal{S}_{t}(C)$ the number of special points living in $C$ at time $t$. It suffices to show that the random time

$$
\tau(C)=\sup \left\{t \geq 0: N_{t}>0\right\}
$$

has finite expectation. Note first that Theorem 7.1 already yields

$$
\mathbb{E} \int_{0}^{\infty} N_{t} \mathrm{~d} t=\int_{0}^{\infty} \mathbb{E} N_{t} \mathrm{~d} t=|C| \int_{0}^{\infty} \beta_{\mathcal{S}_{t}} \mathrm{~d} t<\infty .
$$

Write

$$
N_{t}=N_{0}+N_{t}^{+}-N_{t}^{-},
$$

where $N^{+}$and $N^{-}$are the counting processes of births and deaths of special points in $C$. Since the stochastic intensity of $N^{+}$, say $\lambda_{t}^{N^{+}}$, is

$$
\lambda_{t}^{N^{+}}=\sum_{x \in \mathcal{R}_{t} \cap C} \pi_{\mathcal{S}_{t}}(x)
$$

we have, using the mass transport principle,

$$
\mathbb{E} \lambda_{t}^{N^{+}}=\beta_{\mathcal{R}_{t}}|C| \mathbb{E}_{\mathcal{R}_{t}}^{0} \pi_{\mathcal{S}_{t}}=\beta_{\mathcal{S}_{t}}|C| \mathbb{E}_{\mathcal{S}_{t}}^{0} \pi_{\mathcal{R}_{t}}
$$


Denote the sorted birth and death times of special points in $C$ by $\left(T_{n}^{N^{+}}\right)_{n \geq 1}$ and $\left(T_{n}^{N^{-}}\right)_{n \geq 1}$, respectively. By the definition of stochastic intensity, (7.7), Proposition 6.2 and Theorem 7.1,

$$
\begin{aligned}
\mathbb{E} \sum_{n=1}^{\infty} T_{n}^{N^{+}} & =\mathbb{E} \int_{0}^{\infty} t \mathrm{~d} N_{t}^{+} \\
& =\mathbb{E} \int_{0}^{\infty} t \lambda_{t}^{N^{+}} \mathrm{d} t \\
& =\int_{0}^{\infty} t \mathbb{E} \lambda_{t}^{N^{+}} \mathrm{d} t \\
& \leq|C|\left(\sup _{t} \mathbb{E}_{\mathcal{S}_{t}}^{0} \pi_{\mathcal{R}_{t}}\right) \int_{0}^{\infty} t \beta_{\mathcal{S}_{t}} \mathrm{~d} t<\infty
\end{aligned}
$$

We can write

$$
\int_{0}^{\infty} N_{t} \mathrm{~d} t=\sum_{n=1}^{\infty} T_{n}^{N^{-}}-\sum_{n=1}^{\infty} T_{n}^{N^{+}},
$$

since the last sum is finite by (7.8). Now the claim follows by noting that

$$
\mathbb{E} \tau(C) \leq \mathbb{E} \sum_{n=1}^{\infty} T_{n}^{N^{-}}=\mathbb{E} \int_{0}^{\infty} N_{t} \mathrm{~d} t+\mathbb{E} \sum_{n=1}^{\infty} T_{n}^{N^{+}}<\infty .
$$

\subsection{Coupling from the past}

Throughout this section Assumptions 0-3 are supposed to hold and $\mathcal{Z}_{0}$ is a motion invariant initial condition which satisfies the properties listed in Subsection 3.2.

For each location $y \in \mathbb{R}^{d}$, let $V_{y}$ denote the time it takes for the point process generated by Construct acting on $\Psi_{(0, \infty)}$ with the empty initial condition, and that generated by Construct acting on $\Psi_{(0, \infty)}$ with the initial condition $\mathcal{Z}_{0}$ to couple (i.e. to be identical forever) in the unit ball centered at $y$. Under the foregoing assumptions, the random field $V_{y}$ is translation invariant. From Theorem 7.2, for all $y, \mathbb{E} V_{y}=\mathbb{E} V_{0}<\infty$.

Now, in Theorem 7.2, choose

$$
\mathcal{Z}_{0}:=\text { the nodes of } \Psi_{(-1,0]} \text { alive after running Construct on them, }
$$

as augmentation of the initial condition of Construct acting on $\Psi_{(0, \infty)}$. Let $V_{y}^{(0)}=V_{y}$ denote the associated coupling time field. Consider also the set of nodes of $\Psi_{(-2,-1]}$ still alive at time -1 as augmentation of the initial condition of Construct acting on $\Psi_{(-1, \infty)}$, and denote by $V_{y}^{(1)}$ the associated coupling time field. The random fields $V_{y}^{(0)}$ and $V_{y}^{(1)}+1$ are stochastically equivalent: denoting by $\theta_{t}$ the measure preserving time shift

$$
\theta_{t} \Psi(C \times H)=\Psi(C \times(t+H))
$$


for all Borel sets $C$ of $\mathbb{R}^{d}$ and all Borel sets $H$ of $\mathbb{R}$, we get that

$$
V_{y}^{(0)} \circ \theta_{-1}=V_{y}^{(1)}+1, \quad \forall y .
$$

Continuing like this, we obtain a sequence $\left(V_{y}^{(n)}+n\right)_{n \in \mathbb{N}}$ of identically distributed, and spatially stationary fields.

Lemma 7.3. Under Assumptions 0-3, for all $y \in \mathbb{R}^{d}, V_{y}^{(n)} \rightarrow-\infty$ a.s. when $n \rightarrow \infty$.

Proof. Rewrite $V_{y}^{(n)}$ as $V_{y}^{(n)}+n-n$. Since the sequence $V_{y}^{(n)}+n$ is stationary and ergodic with finite mean, $\frac{V_{y}^{(n)}+n}{n} \rightarrow 0$ when $n \rightarrow \infty$. This implies the announced result.

The following theorem builds a time stationary family of point processes, compatible with the birth and death dynamics.

Theorem 7.3. For $t \in \mathbb{R}$, let $\Phi_{(-n, t)}^{\emptyset}(t)$ denote the point process of nodes that Construct builds alive at time $t$, when starting the dynamics at time $-n$ and with an empty initial condition. For all t, the a.s. limit

$$
\Upsilon_{t}=\lim _{n \rightarrow \infty} \Phi_{(-n, t)}^{\emptyset}(t)
$$

exists and forms a time-stationary family of translation invariant point processes on $\mathbb{R}^{d}$.

Proof. From Lemma 7.3, for all compact sets $C$ of $\mathbb{R}^{d}$, for all $t$, when $n$ tends to $\infty$, $\Phi_{(-n, t)}^{\emptyset}(t)$, couples with a finite random variable $\Upsilon_{t}$ for $n$ larger than a finite random threshold, denoted by $\tau_{t}(C)$, so that the limit (7.9) a.s. exists indeed. The property that $\left(\Upsilon_{t}\right)_{t \in \mathbb{R}}$ is a time-stationary family of point processes follows from the fact that for all $t, \Upsilon_{t}=\Upsilon_{0} \circ \theta_{t}$.

The following theorem completes the analysis of the stationary regimes in terms of convergence in distribution to $\Upsilon_{0}$. We recall that a sequence of point processes $\phi_{n}$ converges in distribution to the point process $\phi$ if and only if for any function $h: \mathbb{R}^{d} \rightarrow \mathbb{R}^{+}$, which has bounded support and is continuous, $\int h d \phi_{n}$ converges in distribution to $\int h d \phi$ in $\mathbb{R}^{+}[12]$.

Theorem 7.4. Let $\Phi_{[0, n)}^{\mathcal{Z}_{0}}(n)$ be the point process of nodes living at time $n$ determined by Construct when starting at time 0 with some initial condition $\mathcal{Z}_{0}$. Under Assumptions 0-3, for all initial conditions $\mathcal{Z}_{0}$ satisfying the assumptions of Subsection 3.2, $\Phi_{[0, n)}^{\mathcal{Z}_{0}}(n)$ converges in distribution to $\Upsilon_{0}$.

Proof. Let us first show that $\Phi_{[0, n)}^{\emptyset}(n)$ converges in distribution to $\Upsilon_{0}$ when $n$ tends to $\infty$. From Theorem 7.3, for all functions $h$ with bounded support $C$ (in particular continuous),

$$
\sup _{A \in \mathcal{B}(\mathbb{R})}\left|\mathbb{P}\left[\int h d \Phi_{[-n, 0)}^{\emptyset}(0) \in A\right]-\mathbb{P}\left[\int h d \Upsilon_{0} \in A\right]\right| \leq \mathbb{P}\left[n<\tau_{0}(C)\right] \rightarrow 0
$$


when $n \rightarrow \infty$. Since $\Phi_{[0, n)}^{\emptyset}(n)=\Phi_{[-n, 0)}^{\emptyset}(0) \circ \theta_{n}$, we can replace $\Phi_{[-n, 0)}^{\emptyset}(0)$ by $\Phi_{[0, n)}^{\emptyset}(n)$ in (7.10), which proves the convergence in distribution of $\Phi_{[0, n)}^{\emptyset}(n)$ to $\Upsilon_{0}$. By arguments similar to those of Theorem 7.3, one gets from Theorem 7.2 that for initial conditions $\mathcal{Z}_{0}$ as above, and for all $C$ compact, $\Phi_{[-n, 0)}^{\emptyset}$ and $\Phi_{[-n, 0)}^{\mathcal{Z}_{0}}$ couple on $C$ for $n$ larger than a finite threshold. This in turn implies that $\Phi_{[-n, 0)}^{\mathcal{Z}_{0}}$ and $\Upsilon_{0}$ couple on $C$ for $n$ larger than a finite threshold. By arguments similar to those above, this finally implies that $\Phi_{[0, n)}^{\mathcal{Z}_{0}}$ converges in distribution to $\Upsilon_{0}$.

\section{Balance equations for moment measures}

The aim of this section is to establish a hierarchy of integral relations between the higher order factorial moment measures of the steady state SBD process $\Upsilon=\Upsilon_{0}$ on $\mathbb{R}^{d}$ constructed in the previous sections. We will denote the factorial moment density of order $k$ by $\rho^{[k]}\left(x_{1}, \ldots, x_{k}\right)$. Notice that $\Upsilon$ is motion invariant (stationary and isotropic). Hence $\rho^{[1]}(x)=\beta$ (the intensity of $\left.\Upsilon\right)$,

$$
\rho^{[2]}(x, y)=\rho_{s t}^{[2]}(x-y)=\rho_{m i}^{[2]}(\|x-y\|)
$$

and for all $k \geq 2$,

$$
\rho^{[k]}\left(x_{1}, \ldots, x_{k}\right)=\rho_{s t}^{[k]}\left(x_{2}-x_{1}, \ldots, x_{k}-x_{1}\right) .
$$

We first establish these balance equations. For $k \geq 2$, the $k$-balance equation relates the $k$-th to the $k-1$-st and the $k+1$-st factorial moment density. For $k=1$, it relates the first and the second densities.

Then we show how to use these equations to get bounds and approximations.

\subsection{Balance equations}

For all $f$ positive, if $\Upsilon=\sum_{n} \delta_{X_{n}}$, we have

$$
\mathbb{E}_{\Upsilon}^{0}\left[\sum_{n \neq 0} f\left(\left\|X_{n}\right\|\right)\right]=\frac{1}{\beta} \int_{\mathbb{R}^{d}} f(\|x\|) \rho_{m i}^{[2]}(\|x\|) d x=\frac{d \nu_{d}}{\beta} \int_{\mathbb{R}^{+}} f(r) \rho_{m i}^{[2]}(r) r^{d-1} d r
$$

with $\nu_{d}$ the volume of the unit ball in $\mathbb{R}^{d}$.

In steady state the mean number of deaths in a Borel set $C$ in the time interval $[0, \epsilon]$ is

$$
\beta|C| \epsilon \mathbb{E}_{\Upsilon}^{0}\left[\sum_{n \neq 0} f\left(\left\|X_{n}\right\|\right)\right]+o(\epsilon)
$$

and it should be equal to the mean number of births in this set and time interval, which is $\lambda|C| \epsilon$. We get from this the following relation:

$$
\int_{\mathbb{R}^{d}} \rho_{m i}^{[2]}(\|x\|) f(\|x\|) d x=\int_{\mathbb{R}^{d}} \rho_{s t}^{[2]}(x) f(\|x\|) d x=\lambda
$$


which is our first balance relation which links the first and the second order factorial moment densities.

Let $C_{1}, C_{2}$ be two Borel sets. Let us look as above at the mean increase (due to births) and the mean decrease (due to deaths) of the following quantity:

$$
\mathbb{E} \sum_{x_{1} \neq x_{2} \in \Upsilon} 1_{C_{1}}\left(x_{1}\right) 1_{C_{2}}\left(x_{2}\right)=\int_{C_{1}} \int_{C_{2}} \rho^{[2]}\left(x_{1}, x_{2}\right) d x_{1} d x_{2} .
$$

The mean increase in an interval of time of length $\epsilon$ is easily seen to be

$$
\lambda \epsilon\left|C_{1}\right| \beta\left|C_{2}\right|+\lambda \epsilon\left|C_{2}\right| \beta\left|C_{1}\right|+o(\epsilon) .
$$

The mean decrease in the same interval is

$$
\begin{aligned}
& \mathbb{E} \sum_{x_{1} \neq x_{2} \in \Upsilon} 1_{C_{1}}\left(x_{1}\right) 1_{C_{2}}\left(x_{2}\right) \epsilon\left(\sum_{z \in \Upsilon, z \neq x_{1}} f\left(\left\|z-x_{1}\right\|\right)+\sum_{z \in \Upsilon, z \neq x_{2}} f\left(\left\|z-x_{2}\right\|\right)\right) \\
+ & o(\epsilon) \\
= & 2 \epsilon \mathbb{E} \sum_{x_{1} \neq x_{2} \in \Upsilon} 1_{C_{1}}\left(x_{1}\right) 1_{C_{2}}\left(x_{2}\right) f\left(\left\|x_{1}-x_{2}\right\|\right) \\
+ & \epsilon \mathbb{E} \sum_{x_{1}, x_{2}, z \in \Upsilon, \text { different }} 1_{C_{1}}\left(x_{1}\right) 1_{C_{2}}\left(x_{2}\right)\left(f\left(\left\|z-x_{1}\right\|\right)+f\left(\left\|z-x_{2}\right\|\right)\right)+o(\epsilon) \\
= & 2 \epsilon \int_{C_{1}} \int_{C_{2}} f\left(\left\|x_{1}-x_{2}\right\|\right) \rho^{[2]}\left(x_{1}, x_{2}\right) d x_{1} d x_{2} \\
+ & \epsilon \int_{C_{1}} \int_{C_{2}} \int_{\mathbb{R}^{d}} f\left(\left\|z-x_{1}\right\|\right)+f\left(\left\|z-x_{2}\right\|\right) \rho^{[3]}\left(x_{1}, x_{2}, z\right) d x_{1} d x_{2} d z+o(\epsilon) .
\end{aligned}
$$

Hence

$$
\begin{aligned}
& 2 \rho^{[2]}\left(x_{1}, x_{2}\right) f\left(\left\|x_{1}-x_{2}\right\|\right)+\int_{\mathbb{R}^{d}} \rho^{[3]}\left(x_{1}, x_{2}, z\right)\left(f\left(\left\|x_{1}-z\right\|\right)+f\left(\left\|x_{2}-z\right\|\right)\right) d z \\
& =2 \beta \lambda
\end{aligned}
$$

that is, for all $x \in \mathbb{R}^{d}$ :

$$
2 \rho_{m i}^{[2]}(\|x\|) f(\|x\|)+\int_{\mathbb{R}^{d}} \rho_{s t}^{[3]}(x, y)(f(\|y\|)+f(\|y-x\|)) d y=2 \beta \lambda,
$$

which is our second balance relation.

The general equation can be obtained in the same way. Let us summarize our findings in:

Theorem 8.1. The factorial moment measures of the time stationary SBD satisfy the following balance relations:

$$
\int_{\mathbb{R}^{d}} \rho_{m i}^{[2]}(\|x\|) f(\|x\|) d x=\lambda
$$


and for all $k \geq 2$, for all $x_{1}, \ldots, x_{k}$ in $\mathbb{R}^{d}$,

$$
\begin{gathered}
\rho^{[k]}\left(x_{1}, \ldots, x_{k}\right)\left(\sum_{i=1, k} \sum_{j=1, k, j \neq i} f\left(\left\|x_{i}-x_{j}\right\|\right)\right) \\
+\int_{\mathbb{R}^{d}} \rho^{[k+1]}\left(x_{1}, \ldots, x_{k}, z\right)\left(\sum_{i=1, k} f\left(\left\|x_{i}-z\right\|\right)\right) d z \\
=\lambda \sum_{i=1, k} \rho^{[k-1]}\left(x_{1}, \ldots, x_{i-1}, x_{i+1}, \ldots, x_{k}\right) .
\end{gathered}
$$

The general relation $(k \geq 2)$ can be re-expressed in terms of the functions $\rho_{s t}^{[k]}$ as

$$
\begin{aligned}
& \rho_{s t}^{[k]}\left(x_{2}-x_{1}, \ldots, x_{k}-x_{1}\right)\left(\sum_{i=1, k} \sum_{j=1, k, j \neq i} f\left(\left\|x_{i}-x_{j}\right\|\right)\right) \\
& +\int_{\mathbb{R}^{d}} \rho_{s t}^{[k+1]}\left(x_{1}-z, \ldots, x_{k}-z\right)\left(\sum_{i=1, k} f\left(\left\|x_{i}-z\right\|\right)\right) d z \\
& =\lambda \sum_{i=2, k} \rho_{s t}^{[k-1]}\left(x_{2}-x_{1}, \ldots, x_{i-1}-x_{1}, x_{i+1}, \ldots, x_{k}-x_{1}\right) \\
& \quad+\lambda \rho_{s t}^{[k-1]}\left(x_{3}-x_{2}, \ldots, x_{k}-x_{2}\right) .
\end{aligned}
$$

\subsection{Bounds and approximations}

Repulsion The next result says that in the stationary regime, there are less points (in terms of their $f$-weight) around a typical point (i.e. under the $\mathbb{P}_{\Upsilon}^{0}$ ) than around a typical location of the Euclidean plane (i.e. under the stationary probability $\mathbb{P}$ ). Note that this $f$-repulsion effect differs from what is usually called repulsion (as in e.g. determinantal point processes).

Theorem 8.2 ( $f$-repulsion). Under the assumptions of Theorem 7.4 , in the stationary regime,

$$
\mathbb{E} \sum_{X \in \Upsilon} f(\|X\|) \geq \mathbb{E}_{\Upsilon}^{0} \sum_{X \in \Upsilon \backslash\{0\}} f(\|X\|)
$$

Proof. From (6.16), in steady state

$$
\lambda a \beta_{\Upsilon}=\beta_{\Upsilon} \mathbb{E}_{\Upsilon}^{0}\left(\pi_{\Upsilon}^{2}\right)
$$

From (6.2), we also have

$$
\lambda=\beta_{\Upsilon} \mathbb{E}_{\Upsilon}^{0}\left(\pi_{\Upsilon}\right)
$$

Hence

$$
a \beta \Upsilon \mathbb{E}_{\Upsilon}^{0}\left(\pi_{\Upsilon}\right)=\mathbb{E}_{\Upsilon}^{0}\left(\pi_{\Upsilon}^{2}\right) \geq\left(\mathbb{E}_{\Upsilon}^{0} \pi_{\Upsilon}\right)^{2},
$$

which directly gives (8.7). 
First Order Approximation It follows from Theorem 8.2 that

$$
\int_{\mathbb{R}^{d}} \rho_{s t}^{[2]}(u) f(\|u\|) d u \leq \beta^{2} \int_{\mathbb{R}^{d}} f(\|u\|) d u=\beta^{2} a .
$$

This and (8.1) give the bound:

$$
\beta \geq \sqrt{\frac{\lambda}{a}}
$$

This can actually be seen as an approximation of order 1 where one (erroneously) pretends that $\rho_{s t}^{[2]}(u)=\beta^{2}$. The first order approximation of the intensity is hence

$$
\widehat{\beta}_{1}=\sqrt{\frac{\lambda}{a}}
$$

for the intensity and

$$
\rho_{1}^{[k]}\left(x_{1}, \ldots, x^{k}\right)=\widehat{\beta}_{1}^{k}
$$

for the $k$-th moment measure.

Second Order Approximation Similarly, let us use the following approximation:

$$
\begin{aligned}
& \int_{\mathbb{R}^{d}} \rho^{[3]}\left(x_{1}, x_{2}, z\right)\left(f\left(\left\|x_{1}-z\right\|\right)+f\left(\left\|x_{2}-z\right\|\right)\right) d z \\
\approx & \frac{1}{\beta} \int_{\mathbb{R}^{d}} \rho^{[2]}\left(x_{1}, x_{2}\right) \rho^{[2]}\left(x_{1}, z\right) f\left(\left\|x_{1}-z\right\|\right) d z \\
+ & \frac{1}{\beta} \int_{\mathbb{R}^{d}} \rho^{[2]}\left(x_{1}, x_{2}\right) \rho^{[2]}\left(x_{2}, z\right) f\left(\left\|x_{2}-z\right\|\right) d z .
\end{aligned}
$$

We then get from this and from (8.2) the (heuristic) equation

$$
\begin{aligned}
& 2 \rho^{[2]}\left(x_{1}, x_{2}\right) f\left(\left\|x_{1}-x_{2}\right\|\right) \\
& \left.\left.+\frac{1}{\beta} \rho^{[2]}\left(x_{1}, x_{2}\right) \int_{\mathbb{R}^{d}}\left(\rho^{[2]}\left(x_{1}, z\right) f\left(\left\|x_{1}-z\right\|\right)\right)+\rho^{[2]}\left(x_{2}, z\right) f\left(\left\|x_{2}-z\right\|\right)\right)\right) d z \\
& \approx 2 \beta \lambda .
\end{aligned}
$$

This gives:

$$
\rho_{m i}^{[2]}(r) \approx \frac{\beta \lambda}{f(r)+\mu}
$$

with

$$
\left.\mu=\frac{1}{\beta} \int_{\mathbb{R}^{d}} \rho_{s t}^{[2]}(x) f(\|x\|)\right) d x .
$$

Multiplying (8.12) by $f(r)$ and integrating leads to the following equation with unknown $\mu$ :

$$
\mu \approx d \nu_{d} \lambda \int_{0}^{\infty} \frac{f(r)}{f(r)+\mu} r^{d-1} d r
$$


The left hand side of the last equation is increasing (in $\mu$ ) from 0 to infinity whereas the right hand side is strictly decreasing from infinity to 0 . Since both functions are continuous, there is one and only one solution to this equation that we will denote by $\widehat{\mu}_{2}$. The second order approximation of the reduced second moment density $g(r)=\frac{1}{\beta} \rho_{m i}^{[2]}(r)$ is then

$$
\widehat{g}_{2}(r)=\frac{\lambda}{f(r)+\widehat{\mu}_{2}}
$$

whereas the second order approximation of the density is

$$
\widehat{\beta}_{2}=\lim _{r \rightarrow \infty} \widehat{g}_{2}(r)=\frac{\lambda}{\widehat{\mu}_{2}} .
$$

and of course

$$
\widehat{\rho}_{m i, 2}^{2]}(r)=\frac{\lambda^{2}}{\widehat{\mu}_{2}\left(f(r)+\widehat{\mu}_{2}\right)} .
$$

Third Order Approximation By the same arguments, the third order approximation is based on the equation

$$
\rho^{[3]}\left(x_{1}, x_{2}, x_{3}\right) \approx \frac{\lambda\left(\rho^{[2]}\left(x_{1}, x_{2}\right)+\rho^{[2]}\left(x_{2}, x_{3}\right)+\rho^{[2]}\left(x_{1}, x_{3}\right)\right)}{2\left(f\left(\left\|x_{1}-x_{2}\right\|\right)+f\left(\left\|x_{2}-x_{3}\right\|\right)+f\left(\left\|x_{3}-x_{1}\right\|\right)\right)+3 \mu},
$$

with $\mu$ as defined above. Let

$$
\begin{aligned}
h\left(x_{1}, x_{2}, z\right) & =\frac{f\left(\left\|x_{1}-z\right\|\right)+f\left(\left\|x_{2}-z\right\|\right)}{2\left(f\left(\left\|x_{1}-x_{2}\right\|\right)+f\left(\left\|x_{1}-z\right\|\right)+f\left(\left\|x_{2}-z\right\|\right)\right)+3 \mu} \\
j\left(x_{1}, x_{2}\right) & =\frac{1}{2 f\left(\left\|x_{1}-x_{2}\right\|\right)+\lambda \int_{\mathbb{R}^{d}} h\left(x_{1}, x_{2}, z\right) d z} .
\end{aligned}
$$

Equations (8.17) and (8.2) lead to the following Volterra type integral equation for the third order approximation of $g^{[2]}\left(x_{1}, x_{2}\right):=\frac{1}{\beta} \rho^{[2]}\left(x_{1}, x_{2}\right)$ :

$$
\begin{aligned}
\widehat{g}_{3}^{[2]}\left(x_{1}, x_{2}\right) & =2 \lambda j\left(x_{1}, x_{2}\right) \\
& -\lambda j\left(x_{1}, x_{2}\right) \int_{\mathbb{R}^{d}}\left(\widehat{g}_{3}^{[2]}\left(x_{1}, z\right)+\widehat{g}_{3}^{[2]}\left(x_{2}, z\right)\right) h\left(x_{1}, x_{2}, z\right) d z .
\end{aligned}
$$

\section{Appendix}

\subsection{Proof of equation (6.2)}

Let $C$ be a bounded Borel set of Lebesgue measure 1. By Proposition 5.1, $\left.\Phi_{(}^{\prime} C\right)$ is integrable. It can be compensated to a martingale by subtracting the continuous process

$$
\int_{0}^{t}\left(\lambda-\sum_{X \in \Phi_{s}^{\prime} \cap C} \pi_{\Phi_{s}^{\prime}}(X)\right) \mathrm{d} s
$$


Taking expectations, using Fubini and the Campbell-Mecke formula we obtain

$$
\begin{aligned}
\beta_{t}-\beta_{0} & =\lambda t-\mathbb{E} \int_{0}^{t} \sum_{X \in \Phi_{s}} \pi_{\Phi_{s}^{\prime}}(X) \mathrm{d} s \\
& =\lambda t-\int_{0}^{t} \mathbb{E} \sum_{X \in \Phi_{s}} \pi_{\Phi_{s}^{\prime}}(X) \mathrm{d} s \\
& =\lambda t-\int_{0}^{t} \beta_{s} E_{\Phi_{s}}^{0} \pi_{\Phi_{s}^{\prime}}(0) \mathrm{d} s
\end{aligned}
$$

from which (6.2) follows by differentiating.

\subsection{Proof of equations (6.19) and (6.27)}

We first recall the general form of the mass transport principle. Let $(\Omega, \mathcal{F}, \mathbb{P})$ be a probability space endowed with a shift $\theta_{u}, u \in \mathbb{R}^{d}$. Let $N$ and $N^{\prime}$ be two $\theta_{u}$-compatible point processes on $\mathbb{R}^{d}$, with respective intensities $\beta_{N}$ and $\beta_{N^{\prime}}$ and Palm probabilities $\mathbb{P}_{0}$ and $\mathbb{P}_{0}^{\prime}$. Then, for all functions $g: \mathbb{R}^{d} \times \Omega \rightarrow \mathbb{R}^{+}$, one has

$$
\beta_{N} \mathbb{E}_{0} \int_{\mathbb{R}^{d}} g(y, \omega) N^{\prime}(d y)=\beta_{N^{\prime}} \mathbb{E}_{0}^{\prime} \int_{\mathbb{R}^{d}} g\left(-x, \theta_{x}(\omega)\right) N(d x) .
$$

We now give the proof for $(6.27)$; $(6.19)$ can be obtained exactly the same way.

The R.H.S. in (6.27) can be rewritten as

$$
\beta_{\mathcal{R}_{t}}|C| \mathbb{E}_{\mathcal{R}_{t}}^{0} \sum_{Y \in \mathcal{Z}_{t}} f(|Y|) \pi_{\mathcal{R}+\mathcal{Z}+\mathcal{A}_{t}}(0)=|C| \beta_{\mathcal{R}_{t}} \mathbb{E}_{\mathcal{R}_{t}}^{0} \int_{\mathbb{R}^{d}} g(y, \omega) \mathcal{Z}_{t}(d y)
$$

with $g(y, \omega)=f(|y|) \pi_{\mathcal{R}+\mathcal{Z}+\mathcal{A}_{t}}(0)$. From the mass transport principle,

$$
\begin{aligned}
\beta_{\mathcal{R}_{t}} \mathbb{E}_{\mathcal{R}_{t}}^{0} \int_{\mathbb{R}^{d}} g(y, \omega) \mathcal{Z}_{t}(d y), & =\beta_{\mathcal{Z}_{t}} \mathbb{E}_{\mathcal{Z}_{t}}^{0} \int_{\mathbb{R}^{d}} g\left(-x, \theta_{x} \omega\right) \mathcal{R}_{t}(d x) \\
& =\beta_{\mathcal{Z}_{t}} \mathbb{E}_{\mathcal{Z}_{t}}^{0} \sum_{X \in \mathcal{R}_{t}} f(|X|) \pi_{\mathcal{R}+\mathcal{Z}+\mathcal{A}_{t} \circ \theta_{X}}(0) \\
& =\beta_{\mathcal{Z}_{t}} \mathbb{E}_{\mathcal{Z}_{t}}^{0} \sum_{X \in \mathcal{R}_{t}} f(|X|) \pi_{\mathcal{R}+\mathcal{Z}+\mathcal{A}_{t}}(X)
\end{aligned}
$$

The L.H.S. in (6.27) can be rewritten as

$$
\begin{aligned}
\mathbb{E} \sum_{Y \in \mathcal{Z}_{t} \cap C} & \sum_{X \in \mathcal{R}_{t}} f(|X-Y|) \pi_{\mathcal{R}+\mathcal{Z}+\mathcal{A}_{t}}(X) \\
= & \mathbb{E} \sum_{Y \in \mathcal{Z}_{t} \cap C} \sum_{X \in \mathcal{R}_{t}} f(|X-Y|) \pi_{\mathcal{R}+\mathcal{Z}+\mathcal{A}_{t} \circ \theta_{Y}}(X-Y) \\
= & \beta_{\mathcal{Z}_{t}}|C| \mathbb{E}_{\mathcal{Z}_{t}}^{0} \sum_{X \in \mathcal{R}_{t}} f(|X|) \pi_{\mathcal{R}+\mathcal{Z}+\mathcal{A}_{t}}(X),
\end{aligned}
$$

which concludes the proof. 


\subsection{Table of notation}

\begin{tabular}{|c|c|}
\hline$a=\int_{\mathbb{R}^{d}} f(\|x\|) d x$ & Strength of the response function \\
\hline $\mathcal{A}$ & Set of A points \\
\hline $\mathcal{A}(z)$ & Set of A points offspring of $z$ \\
\hline$b_{p}$ & Birth time of point $p$ \\
\hline$\beta_{X}$ & Intensity of the stationary point process $X$ on $\mathbb{R}^{d}$ \\
\hline$B(x, r)$ & Ball of radius $r$ centered in $x$ \\
\hline$d$ & Dimension of the Euclidean space \\
\hline Construct & Pathwise construction of the set of nodes living at all $t$ \\
\hline Construct $^{Z}$ & $\begin{array}{l}\text { Simultaneous construction of the set of nodes living at } \\
\text { all } t \text {, for two different initial conditions }\end{array}$ \\
\hline$D$ & Convex set in $\mathbb{R}^{d}$ \\
\hline$d_{p}$ & Death time of point $p$ \\
\hline$\delta_{x}$ & Dirac measure at $x$ \\
\hline $\mathbb{E}_{\chi}^{0}$ & Palm probability of the $\chi$ point process \\
\hline$f: \mathbb{R}^{+} \rightarrow \mathbb{R}^{+}$ & Response function \\
\hline$I_{p q}$ & Connection direction for $(p, q)$ \\
\hline IS & Investigation stack \\
\hline$K$ & Upper-bound on $f$ \\
\hline$\lambda$ & Birth rate \\
\hline$l^{d}(C)=|C|$ & Lebesgue measure on $\mathbb{R}^{d}$ of the Borel set $C$ \\
\hline$\mu$ & Death rate \\
\hline$\nu_{d}$ & volume of a unit ball \\
\hline$\Phi_{t}$ & $\begin{array}{l}\text { Counting measure of the nodes living at time } t \text { for the } \\
\emptyset \text { initial condition as obtained by Construct }\end{array}$ \\
\hline$\Phi_{t}^{\prime}$ & $\begin{array}{l}\text { Counting measure of the nodes living at time } t \text { for the } \\
\mathcal{Z} \text { initial condition as obtained by Construct }\end{array}$ \\
\hline$\widetilde{\Phi}_{t}$ & $\begin{array}{l}\text { Counting measure of the nodes unfinished at time } t \text { as } \\
\text { obtained by Construct }\end{array}$ \\
\hline$\pi_{X}(z)=\sum_{x \in X} f(|x-z|)$ & Death pressure exerted on $z$ by the point process $X$. \\
\hline$\Pi_{X}(Y)=\sum_{y \in Y} \pi_{X}(y)$ & $\begin{array}{l}\text { Death pressure exerted on the point process } Y \text { by the } \\
\text { point process } X\end{array}$ \\
\hline$p, q, \ldots$ & Points of $\Psi$ \\
\hline$\Psi$ & Arrival counting measure on $\mathbb{R} \times \mathbb{R}^{d}$ \\
\hline$\Psi_{t}$ & Arrival counting measure on $\mathbb{R} \times \mathbb{R}^{d}$ after time $t$ \\
\hline$\Psi_{(s, t)}$ & Arrival counting measure on $\mathbb{R} \times \mathbb{R}^{d}$ in interval $(s, t)$ \\
\hline $\mathcal{R}$ & Set of regular points \\
\hline $\mathrm{RCG}$ & Random Connection Graph \\
\hline SBD & Spatial Birth and Death \\
\hline$S_{p}$ & Stack of node $p$ \\
\hline $\mathcal{S}$ & Set of special points \\
\hline
\end{tabular}




\begin{tabular}{|l|l|}
\hline $\mathcal{S}(z)$ & Set of special points offspring of $z$ \\
\hline$t$ & Time \\
\hline$T_{p q}$ & Connection time for $(p, q)$ \\
\hline$x, y, \ldots$ & Points of $\Phi$ \\
\hline$x_{p}$ & Birth location of point $p$ \\
\hline $\mathcal{Z}$ & Set of Z points \\
\hline $\mathcal{Z}_{0}$ & Initial condition point process \\
\hline $\mathcal{Z}(z)$ & Set of Z points offspring of $z$ \\
\hline $\mathfrak{z}(s)$ & Ancestor of the special node $s$ \\
\hline
\end{tabular}

\section{Acknowledgements}

The authors thank Mayank Manjrekar and the two reviewers for their comments on the paper.

The research of the first author was supported by an award from the Simons Foundation (\# 197982 to The University of Texas at Austin). The work of the third author was supported by the Academy of Finland with Senior Researcher funding and by EIT ICT Labs through the project DCDWN. The work presented in this paper has been partly carried out at LINCS (http://www.lincs.fr).

\section{References}

[1] F. Baccelli, F. Mathieu, I. Norros, and R. Varloot, "Can p2p networks be superscalable?" in Proceedings of IEEE INFOCOM 2013, Torino, Italy, April 2013.

[2] D.J. Daley and D. Vere-Jones, An Introduction to the Theory of Point Processes. Springer, 1988.

[3] D.L. Finkelshtein and Yu.G. Kondratiev, "Regulation mechanisms in spatial stochastic development models", J. Stat. Phys. 136(1), 103-115, 2009.

[4] D.L. Finkelshtein, Yu.G. Kondratiev and O. Kutoviy, "Semigroup approach to birth-and-death stochastic dynamics in continuum", J. Funct. Anal. 262(3), 12741308, 2012.

[5] D.L. Finkelshtein, Yu.G Kondratiev, Y. Kozitsky and O. Kutoviy, "Stochastic evolution of a continuum particle systems with dispersal and competition", Eur. Phys. J. Special Topics, Number 216, 107-116, 2013.

[6] D.L. Finkelshtein, Yu.G Kondratiev, and M. J. Oliveira, " Markov evolutions and hierarchical equations in the continuum. I. One-component systems". J. Evol. Equ. 9(2), 197-233, 2009. 
[7] M. Franceschetti and R. Meester, Random Networks for Communications. CUP, 2007.

[8] N. Garcia, "Birth and death processes as projections of higher dimensional Poisson processes", Adv. in Appl. Probab. 1(4), 911-930, 1995.

[9] N. Garcia and T. Kurtz, "Spatial birth and death processes as solutions of stochastic equations", ALEA Lat. Am. J. Probab. Math. Stat. 1, 281-303, 2006.

[10] N. Garcia and T. Kurtz, "Spatial Point Processes and the Projection Method", in In and Out of Equilibrium 2, vol. 60 of the series Progress in Probability, 271-298, Birkhäuser, Basel, 2008.

[11] R.A. Holley and D.W. Stroock, " Nearest neighbor birth and death processes on the real line", Acta Math. 140(1-2), 103-154, 1978.

[12] O. Kallenberg, Random measures. Berlin: Akademie-Verlag, 1976.

[13] Yu.G. Kondratiev and Y. Kozitsky, "Evolution of states in a continuum migration model", arXiv:1607.05871, 2016.

[14] Yu.G. Kondratiev, O. Kutoviy and R. Minlos, "On non-equilibrium stochastic dynamics for interacting particle systems in continuum", J. Funct. Anal. 255, 200-227, 2008.

[15] G. Last and H. Thorisson, "Invariant transports of stationary random measures and mass-stationarity," Ann. Probab. 37(2), 790-813, 2009.

[16] S. Meyn and R. Tweedie, Markov Chains and Stochastic Stability. Springer Verlag, 1993.

[17] J. Møller and R. P. Waagepetersen, Statistical inference and simulation for spatial point processes, volume 100 of Monographs on Statistics and Applied Probability, Chapman \& Hall/CRC, Boca Raton, FL, 2004. ISBN 1-58488-265-4.

[18] M. D. Penrose, "On a continuum percolation model", Adv. Appl. Probab. 23(3), 536???556, 1991.

[19] M. D. Penrose, "Existence and spatial limit theorems for lattice and continuum particle systems", Probability Surveys 5, 1-36, 2008.

[20] C. Preston, "Spatial birth-and-death processes", Bull. Inst. Internat. Statist. 46(2), 371-391, 1975.

[21] D. Stoyan, W. S. Kendall, and J. Mecke, Stochastic Geometry and its Applications, 2nd ed. Chichester: Wiley, 1995. 